DOI: $10.24850 /$ j-tyca-2022-02-07

Artículos

\title{
Estimación de la distribución espacio temporal de la recarga de agua subterránea en regiones húmedas con clima tropical
}

\section{Estimation of spatiotemporal groundwater recharge distribution in humid regions with tropical climate}

Marcia Lizeth Barrera-de-Calderón1, ORCID: https://orcid.org/00000001-7627-150X Jaime Garfias², ORCID: https://orcid.org/0000-0001-6388-2109

Richard Martel3, ORCID: https://orcid.org/0000-0003-4219-5582

Javier Salas-García4, ORCID: https://orcid.org/0000-0002-1297-7893

1 Universidad Autónoma del Estado de México-Universidad de El Salvador San Salvador, El Salvador, marcia.barrera@ues.edu.sv

2Instituto Interamericano de Tecnología y Ciencias del Agua (IITCA), Universidad Autónoma del Estado de México (UAEM), Toluca, Estado de México, México, jgarfiass@gmail.com 
${ }^{3}$ Institut National de la Recherche Scientifique (INRS Ete), Universidad de Quebec, Quebec, Canadá, richard.martel@ete.inrs.ca

${ }^{4}$ Facultad de Ingeniería, Universidad Autónoma del Estado de México (UAEM), Toluca, Estado de México, México, proyectos@javiersalasg.com

Autora para correspondencia: Marcia Lizeth Barrera-de-Calderón, marcia.barrera@ues.edu.sv

\section{Resumen}

La determinación precisa de la variación de la recarga de agua subterránea es una tarea fundamental para la planificación sostenible de los recursos hídricos subterráneos, sobre todo en acuíferos fuertemente presionados. Con el objetivo de determinar la variabilidad espacial y temporal de la recarga de agua subterránea en un acuífero urbano con clima húmedo, como el acuífero de San Salvador, se utilizaron dos métodos de balance de masas: uno en la zona subsuperficial, balance de humedad del suelo (SWB, por sus siglas en inglés de soil water balance) y otro en la zona saturada, balance de masa de cloruros (CMB, por sus siglas en inglés de chloride mass balance). EI SWB fue calculado a escala diaria para cuatro años (2012-2015) mediante el método de Thornthwaite y Mather modificado, utilizando un conjunto de mallas de datos climáticos diarios y datos físicos de la zona de estudio. El CMB se empleó para determinar la recarga de agua subterránea de la zona saturada en 
muestras tomadas en pozos y manantiales durante los años 2009 y 2016 en la parte alta de la cuenca, donde diversos estudios sugieren que ocurre la principal recarga del acuífero. Los resultados del SWB indican una fuerte variación temporal y espacial de la recarga en la zona de estudio, que puede variar entre 326 y 561 mm año-1 en años secos y húmedos, respectivamente. Los resultados del CMB mostraron consistencia con el SWB; los valores de recarga oscilaron entre 313 y $693 \mathrm{~mm}$ año-1. En ambos métodos, la recarga media anual es similary representa entre el 20 y $30 \%$ de la precipitación. La aplicación conjunta de ambos métodos podría utilizarse en zonas similares; su elección dependerá de los objetivos del estudio.

Palabras clave: recarga de agua subterránea, balance de humedad de suelo (SWB), balance de masa de cloruros (CMB), acuífero urbano, acuífero de San Salvador.

\section{Abstract}

The precise determination of groundwater recharge variation is a fundamental task for the sustainable planning of groundwater resources, particularly in heavily pressured aquifers. In order to determine the spatial and temporal variability of groundwater recharge in an urban aquifer with a humid climate, such as the San Salvador aquifer, two Mass Balance methods were used (one in the subsurface zone, Soil Water Balance (SWB) and another in the saturated zone, Chloride Mass Balance 
(CMB)). The SWB was calculated on a daily scale for four years (20122015) through the modified Thornthwaite and Mather method, using a set of daily climatic data grids and physical data from the study area. The CMB was used to determine the groundwater recharge in drilled wells and springs samples taken during 2009 and 2016, in the upper part of the basin, where various studies suggest that the main aquifer recharge occurs. The results of the SWB indicate a strong temporal and spatial variation of the recharge in the study area, which can vary between 326 and $561 \mathrm{~mm}$ year $^{-1}$, in dry and wet years, respectively. The CMB results showed consistency with the SWB, groundwater recharge values ranged between 313 and $693 \mathrm{~mm}_{\text {year }}{ }^{-1}$. In both methods the mean annual recharge is similar and represents between $20 \%$ and $30 \%$ of the precipitation. The application of both methods could be used in similar areas, the selection of the method will depend on the objectives of the study.

Keywords: Groundwater recharge, soil water balance (SWB), chloride mass balance (CMB), urban aquifer, San Salvador aquifer.

Recibido: 08/01/2021

Aceptado: 03/04/2021 
Tecnología y

Ciencias Agua
2022, Instituto Mexicano de Tecnología del Agua

Open Access bajo la licencia CC BY-NC-SA 4.0

(https://creativecommons.org/licenses/by-nc-sa/4.0/)

\section{Introducción}

La recarga acuífera, definida como el agua que se mueve desde la zona no saturada e ingresa en la zona saturada (Heppner, Nimmo, Folmar, Gburek, \& Risser, 2007), es esencial para el estudio de los recursos hídricos (Scanlon, Healy, \& Cook, 2002; Lihe et al., 2011). Su estimación es indispensable para una apropiada planificación y gestión de los sistemas de agua subterránea (Healy \& Cook, 2002) en la cuenca hidrológica. Sin embargo, debido a la dificultad de medirla directamente y a la incertidumbre asociada debe ser cuantificada indirectamente por distintos métodos (Scanlon et al., 2002; Heppner et al., 2007; Lihe et al., 2011), esperando consistencia en los resultados, aunque ello no implique precisión (Healy \& Cook, 2002).

La estimación de la recarga natural es compleja (Sophocleous, 1993; Healy \& Cook, 2002; Lerner, 2002; Scanlon et al., 2002), sobre todo cuando se conoce de manera muy limitada el funcionamiento del acuífero, y se requiere cuantificar su variabilidad espacial y temporal (Scanlon et al., 2002). Con frecuencia los modeladores, planificadores y diseñadores de políticas para el manejo de acuíferos obvian la variabilidad de la recarga y asumen un valor promedio para toda la cuenca, la cual es estimada como una fracción de la precipitación o es ajustada como 
parámetro de calibración en un modelo de simulación numérica (Dripps \& Bradbury, 2009).

La cuantificación de la recarga implica la recarga directa, la recarga puntual y la recarga indirecta (Lerner, 2002). Debido a la complejidad en la determinación de la recarga directa o natural (utilizados como sinónimos en este documento), varios autores, entre ellos, Lerner (1990), Scanlon et al. (2002), Lerner (2002), Healy y Cook (2002), y Doble y Crosbie (2017) enfatizan la necesidad del uso de varias técnicas para su estimación, reduciéndose así la incertidumbre. La aplicación de las técnicas depende principalmente de su variación espacial y temporal, la disponibilidad de información, clima predominante, tasa de recarga, periodo de tiempo representado y extensión superficial en la cual se realizan los estudios (Lerner, 1990; Lerner, 2002; Scanlon et al., 2002; Doble \& Crosbie, 2017). Estas técnicas son explicadas en detalle por Scanlon et al. (2002), Lerner (2002), Seiler y Gat (2007), y Healy y Scanlon (2010).

Un método físico de la zona subsuperficial muy utilizado en climas húmedos es el balance de humedad de suelo (SWB) (Thornthwaite, 1948; Thornthwaite \& Mather, 1955; Thornthwaite \& Mather, 1957). El procedimiento se enfoca en establecer el balance de agua para la zona radicular. Según Steenhuis y Van-Der-Molen (1986), el SWB ha sido aplicado con éxito para balances de agua a nivel de cuenca y para estimar la recarga de agua subterránea. Una de sus principales ventajas es el uso de información climática y geográfica comúnmente disponible. Sin 
embargo, en muchas ocasiones, principalmente en países en desarrollo, la escala de cálculo temporal es mensual, lo cual puede llegar a subestimar los resultados hasta un $25 \%$ con respecto a estimaciones diarias (Rushton \& Ward, 1979).

Varios estudios (Dripps \& Bradbury, 2007; Dripps \& Bradbury, 2009; Stanton, Ryter, \& Peterson, 2012; Davis \& Putnam, 2013; Mair et al., 2013; Cao, Scanlon, Han, \& Zheng, 2016; Day \& Simpkins, 2018, entre otros) han determinado la variabilidad espacial y temporal de la recarga de agua subterránea con el uso del SWB incorporando la variabilidad espacial y temporal de la recarga mediante programas estructurados cuyos módulos utilizan sistemas de información geográfica. De forma más reciente, otros autores (Izuka et al., 2018; McLean et al., 2019) han manejado gran cantidad de datos con la incorporación del formato NetCDF para modelos de recarga diaria de varios años con el SWB (Westenbroek, Engott, Kelson, \& Hunt, 2018), facilitando la visualización y el manejo de las series temporales de recarga.

El uso de trazadores químicos en la zona saturada también ha sido ampliamente utilizado para estimar la recarga de agua subterránea, en especial los cloruros, debido a su bajo costo y facilidad para determinarlos y cuantificar la recarga. El método del balance de masa de cloruros (CMB) fue propuesto por Eriksson y Khunakasem (1969), y ha sido utilizado por distintos investigadores para cuantificar la recarga de agua subterránea. Por ejemplo, Edmunds y Gaye (1994) lo utilizaron en la zona intersticial y en pozos someros en un extenso acuífero costero de Senegal; por su 
parte, Ng, McLaughlin, Entekhabi y Scanlon (2010) lo emplearon en la evaluación de los efectos del cambio climático en la recarga mediante mediciones de cloruros en la zona no saturada.

Mientras tanto, Lihe et al. (2011) cuantificaron la tasa de recarga para Ordos Plateu, China; Crosbie, McCallum, Walker y Chiew (2012) lo utilizaron como parámetro de comparación de un modelo para evaluar la recarga episódica (pulsos irregulares e infrecuentes de recarga de agua subterránea según Lewis y Walker (2002)) alterada por los efectos del cambio climático en el acuífero High Plains, EUA. Por su parte, MarreroDiaz et al. (2015) determinaron la recarga neta total para un año húmedo en el acuífero volcánico Las Cañadas, Islas Canarias; Ifediegwu (2020) lo usó en una zona del estado de Enugu, Nigeria, caracterizada por clima húmedo; mientras que Nemaxwi, Odiyo y Makungo (2019) lograron presentar la distribución espacial de la recarga a partir de determinaciones puntuales de recarga en un acuífero cuaternario en la provincia de Limpopo, Sudáfrica.

En el acuífero de San Salvador, la distribución espacial y temporal de las tasas de recarga de agua subterránea aún se entiende muy poco. Tradicionalmente, su estimación ha sido con el método de balance de humedad de suelo a escala mensual sobre una base de datos climáticos multianuales, y en un ámbito espacial que agrupa características físicas y climáticas del territorio (Servicio Hidrológico Nacional, 2005; MARN, 2016a). Este análisis proporciona valores medios de la recarga que desestima su variación en el espacio y el tiempo, lo cual es principalmente 
preocupante cuando se trata de la recarga para años secos, donde la recarga puede disminuir de modo considerable. Además, los cálculos existentes aún no han sido contrastados con metodologías alternativas que aumenten la confiabilidad de los resultados. Esto podría llegar a impactar de forma negativa en la planificación sostenible del acuífero.

El objetivo de este estudio es determinar la variabilidad espacial y temporal de la recarga natural de agua subterránea en el acuífero urbano de San Salvador para el periodo 2012-2015, que contempla años húmedos y secos, mediante el SWB en un paso de tiempo diario y en una red discreta definida para la zona. Los datos son contrastados con estimaciones de la recarga realizados mediante el CMB para dos años: 2008 y 2015 . Con los resultados se pretende proporcionar información consistente y más confiable a los tomadores de decisión para el desarrollo sostenible del acuífero que abastece el $41 \%$ del área metropolitana de San Salvador y alertar sobre la variabilidad de la oferta de agua subterránea para tomar decisiones conscientes respecto a su explotación. Este estudio puede ser replicado en acuíferos con similares condiciones.

\section{EI acuífero de San Salvador: características climáticas, físicas y antrópicas que influencian el balance de agua subterránea}


El acuífero de San Salvador suministra alrededor del $42 \%$ de la demanda de agua de la región más densamente poblada de El Salvador (Digestyc, 2008): la región metropolitana de San Salvador (AMSS); se localiza en el subsuelo de dicha zona, en la subcuenca alta del río Acelhuate, cuya área es de unos $362 \mathrm{~km}^{2}$ (Figura 1). La zona se caracteriza por ser muy accidentada al sur y presentar menores pendientes al norte; sus elevaciones varían de 1917 a 359 msnm. Posee un clima tropical con dos estaciones de seis meses cada una, una seca y una lluviosa; temperaturas medias anuales de $16.3^{\circ} \mathrm{C}$ en la zona alta y $23.4{ }^{\circ} \mathrm{C}$ en la zona baja; la precipitación media registrada varía de $2141 \mathrm{~mm}$ año-1 en la parte alta a $1731 \mathrm{~mm}^{2}$ ño-1 en la parte baja (MARN, 2016b). 
Tecnología y

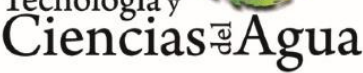

2022, Instituto Mexicano de Tecnología del Agua

Open Access bajo la licencia CC BY-NC-SA 4.0

(https://creativecommons.org/licenses/by-nc-sa/4.0/)
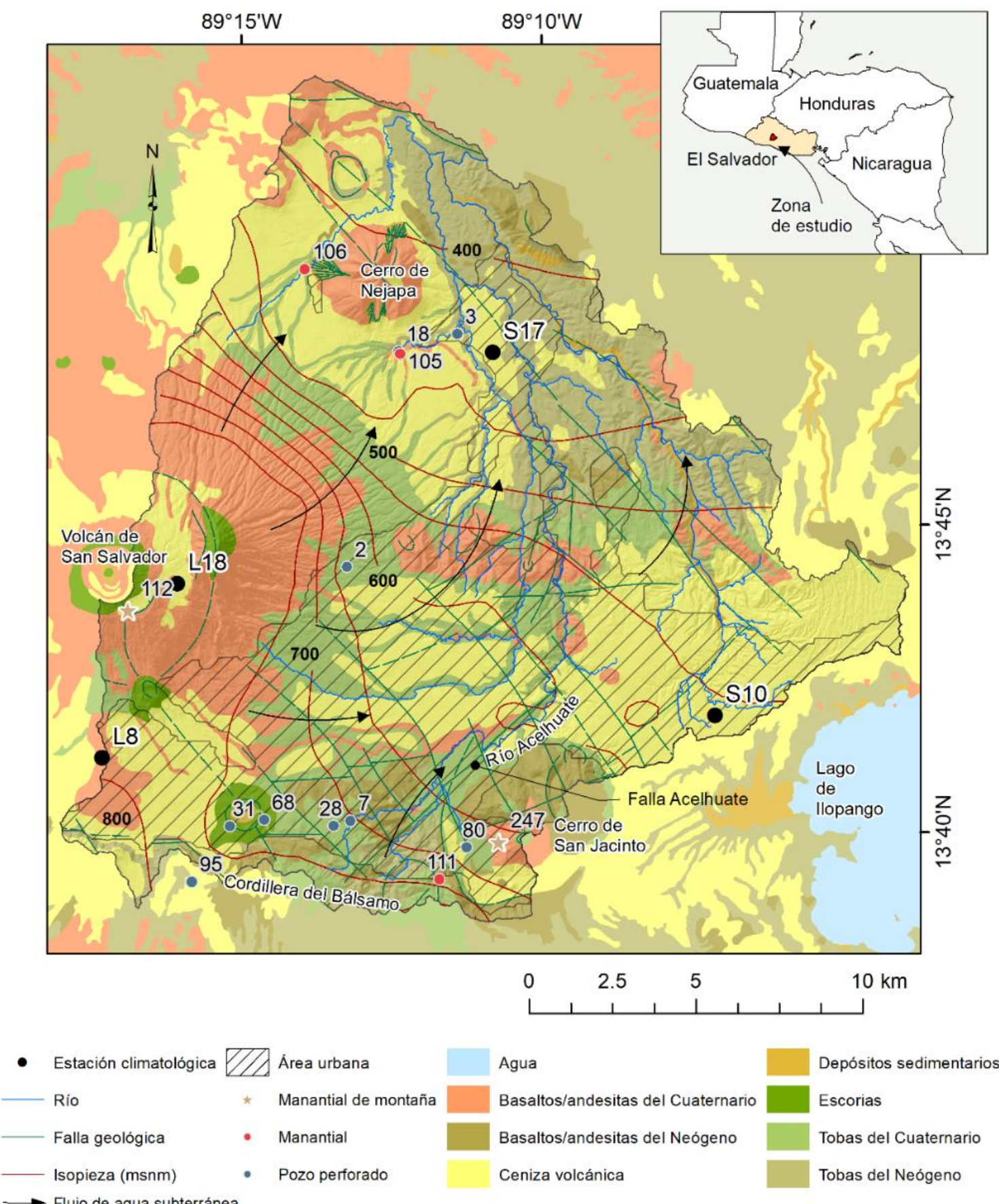
Figura 1. Ubicación de la zona de estudio mostrando la geología superficial simplificada, nivel piezométrico y dirección de flujo, estaciones pluviométricas, pozos y manantiales muestreados para el método CMB (Ios números indican el código utilizado en este estudio) y principales accidentes geográficos.

De acuerdo con MARN y OPAMSS (2012), en la zona de estudio predominan las zonas urbanas, comerciales e industriales (41.65\%); café y bosques siempre verdes (22.24\%); granos básicos y cultivos asociados (18.46\%); frutales y monocultivos (13\%); el resto es utilizado por vegetación herbácea, playas y zonas de extracción de arena. En adición a lo anterior, la textura del suelo en la zona es predominantemente franca, lo cual favorece la infiltración. De acuerdo con Rico, Bourne y Menéndez (1965), el $63 \%$ posee materiales franco arenosos, coincidiendo con las zonas altas y vegetadas, así como con gran parte de la zona urbana; el $19 \%$ corresponde a materiales francos, al pie del volcán de San Salvador y al norte de la zona de estudio; el $13 \%$ son materiales franco limosos, localizados al norte de la zona de estudio, y el $5 \%$ restante es material de menor permeabilidad, que aparece cubriendo accidentes geográficos como el cerro de Nejapa (al norte) y las faldas del cerro de San Jacinto (al sur) (Figura 1). 


\section{Contexto geológico, estructural e hidrogeológico}

El área de interés se originó como producto de la actividad tectónica, volcánica y erosiva de la región, entre el Neógeno y el Cuaternario. A raíz de la intensa actividad del Arco Volcánico Centroamericano (CAVA) (Carr \& Stoiber, 1977), tres formaciones, en orden de antigüedad, afloran en la zona: Bálsamo, Cuscatlán y San Salvador (Williams \& Meyer-Abich, 1955; Weber Wisemann, \& Wittekindt, 1974; Reynolds, 1980); todas consisten en interestratificaciones de piroclástos, tobas, y lavas basálticas y andesíticas, originadas sobre todo como producto de los materiales eyectados o erupcionados del estratovolcán de San Salvador, el nuevo volcán Boquerón, y las calderas de Ilopango y Coatepeque (SchmidtThomé, 1975; Lexa, Šebesta, Chavez, Hernández, \& Pécskay, 2012; Hernández \& Jicha, 2019) (Figura 1).

Algunos autores (PNUD, 1972; ANDA, 2008; Barrera-de-Calderón, Garfias, Martel, \& Salas-García, 2021a) han determinado que la zona más productiva del acuífero se localiza al sur del área de estudio y coincide con la presencia de lavas fracturadas interestratificadas con piroclastos; en esas zonas, la conductividad hidráulica puede alcanzar $10^{3} \mathrm{~m}^{\mathrm{d}} \mathrm{d}^{-1}$. Mientras que la mayor extensión y espesor del acuífero corresponde con piroclastos y tobas, en los cuales las conductividades hidráulicas oscilan 
Tecnología y

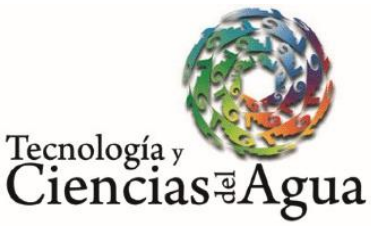

2022, Instituto Mexicano de Tecnología del Agua

Open Access bajo la licencia CC BY-NC-SA 4.0

(https://creativecommons.org/licenses/by-nc-sa/4.0/)

entre $10^{-2}$ y $1 \mathrm{~m}^{-\mathrm{d}^{-1}}$ (un mapa de distribución de conductividades hidráulicas en la zona de estudio puede ser consultado en Barrera-deCalderón et al., 2021a).

En ese contexto hidrogeológico, se han identificado dos sistemas principales de flujo (Barrera-de-Calderón, 2010; Barrera-de-Calderón et al., 2021a): el primero circula radialmente desde el volcán de San Salvador y luego se conduce hacia el norte, en una tendencia similar al recorrido del río Acelhuate; el segundo se mueve inicialmente al noreste y luego hacia el norte, para converger con el primero en la salida de la subcuenca.

Además, autores como PNUD (1972); MARN (2016a) y Barrera-deCalderón (2010) han sugerido que las principales zonas de recarga en la zona de estudio se encuentran en las laderas del volcán de San Salvador; mientras que las principales descargas naturales han sido identificadas a lo largo de la falla Acelhuate en forma de manantiales, en el cambio de conductividad hidráulica entre los flujos de lava fracturada y los piroclastos (Barrera-de-Calderón et al., 2021a).

Asimismo, la hidrogeoquímica del acuífero ha permitido confirmar que la principal fuente de recarga de agua subterránea corresponde a la precipitación, la cual, debido a su elevada importancia, es calculada en este estudio mediante el SWB y CMB. Sin embargo, existe un aporte de recarga urbana procedente de fugas en los sistemas de agua potable y alcantarillado de la ciudad que debe tenerse en cuenta, en especial por 
sus efectos en la calidad del agua y el balance hidrológico (Barrera-deCalderón, Garfias, Martel, \& Salas-García, 2021b). Para evitar el efecto de dicha recarga en el CMB, se eligieron aquellos pozos localizados aguas arriba de las zonas urbanas, en los límites entre dicha zona y el área vegetada. En estos pozos se consideró que la afectación del urbanismo, si existiese, es tan pequeña comparada con la recarga natural, que puede despreciarse.

\section{Métodos y modelos utilizados}

\section{Balance de humedad del suelo (SWB 2.0)}

El modelo de balance de humedad de suelo (SWB 2.0) (Westenbroek et al., 2018) se empleó para estimar la recarga natural potencial de agua subterránea, cuantificada en este artículo como la infiltración neta que pasa la zona radicular en el subsuelo (Dripps \& Bradbury, 2007). El SWB 2.0 es un modelo determinístico, cuasi tridimensional, basado en datos 
físicos del suelo y climáticos para la zona de estudio (Dripps \& Bradbury, 2009), el cual utiliza el método de Thornthwaite-Matter modificado para tener en cuenta la humedad del suelo.

Está integrado por tres reservorios que almacenan agua, denominados nieve, intercepción y suelo. Para el caso de climas tropicales, el reservorio de nieve no es considerado, mientras que el reservorio de intercepción acumula agua interceptada diariamente por el dosel de los árboles y la vegetación, previo a su contacto con el suelo. La variación diaria en la humedad del suelo es modelada en el reservorio de suelo para el dominio del modelo discretizado mediante celdas de un ráster con profundidad igual a la zona radicular mediante la Ecuación (1). En esta ecuación se consideran los ingresos y salidas de humedad al reservorio; asimismo, se considera la humedad del día antecedente como una condición inicial para el día de simulación:

$H S=P-I n t-E S C-E T+H S_{i}$

Donde HS representa la humedad del suelo para el día de simulación en $\mathrm{mm} ; P$, la precipitación en $\mathrm{mm}$; Int, la intercepción de la vegetación $(\mathrm{mm})$; Esc, la escorrentía superficial en $\mathrm{mm}$; $E T$, la evapotranspiración real en $\mathrm{mm}$, y $H S_{i}$ representa a la humedad del suelo en el día previo a la simulación en mm. 
Se asume que la humedad del suelo oscila entre el punto de marchitez permanente y la capacidad de campo, que varían con la textura del suelo. La diferencia entre ambos valores corresponde a la capacidad de agua disponible en el suelo. El total de agua disponible para el suelo se calcula con la Ecuación (2). Cada vez que la humedad del suelo supera este valor, se considera que un evento de recarga de agua subterránea ha ocurrido en esa celda:

$T A W=\left(H S_{c c}-H S_{P M P}\right) \cdot P R$

Donde TAW es la capacidad total de agua disponible $(\mathrm{mm}) ; H S_{C C}$, la humedad del suelo a capacidad de campo $\left(\mathrm{mm} \mathrm{m}^{-1}\right)$; HSPMP, la humedad de suelo en el punto de marchitez permanente $\left(\mathrm{mm} \mathrm{m}^{-1}\right)$, y $P R$ corresponde a la profundidad efectiva de raíces de la vegetación (m).

En el modelo de recarga del acuífero de San Salvador, el cálculo de la humedad del suelo para cada día de simulación en cada celda del modelo requirió de un conjunto de datos ráster diarios de lluvia, y temperatura máxima y mínima, dirección de drenaje superficial, así como uso y textura del suelo para los módulos de escorrentía, evapotranspiración e intercepción, según el caso. Asimismo, los ráster textura y uso de suelo se usaron para calcular la capacidad total de agua disponible. 
Asimismo, los métodos, opciones de cálculo, y especificaciones de mallas de entrada y salida para el modelo de recarga se codificaron mediante un archivo de control, complementado con una tabla de búsqueda mediante la cual se relacionaron los archivos ráster y los valores de los parámetros relativos al uso y textura de suelo.

Los datos crudos de lluvia y temperatura diaria se obtuvieron de los registros del Ministerio de Medio Ambiente y Recursos Naturales (MARN); presentaban datos faltantes, principalmente debido a fallas en el equipo o errores de medición, y por lo tanto se requirió un proceso de relleno de datos. El relleno de las series de precipitación se realizó para el periodo 2012-2015 (cuatro años que presentaron la menor cantidad de datos faltantes).

Para el rellenado de los datos se utilizó una regresión lineal simple para la estación L8 Procafé, y una regresión lineal múltiple para las estaciones S04 Apopa y L18 Boquerón (Figura 1 y Figura 2), donde se obtuvieron las correlaciones más altas. La estación base para completar las series fue la estación Ilopango S10, la cual cuenta con uno de los registros más completos que tiene el país sobre precipitación y temperatura para el periodo 1970-2015. 
Tecnología y

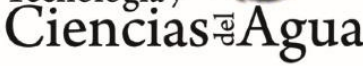

2022, Instituto Mexicano de Tecnología del Agua

Open Access bajo la licencia CC BY-NC-SA 4.0

(https://creativecommons.org/licenses/by-nc-sa/4.0/)
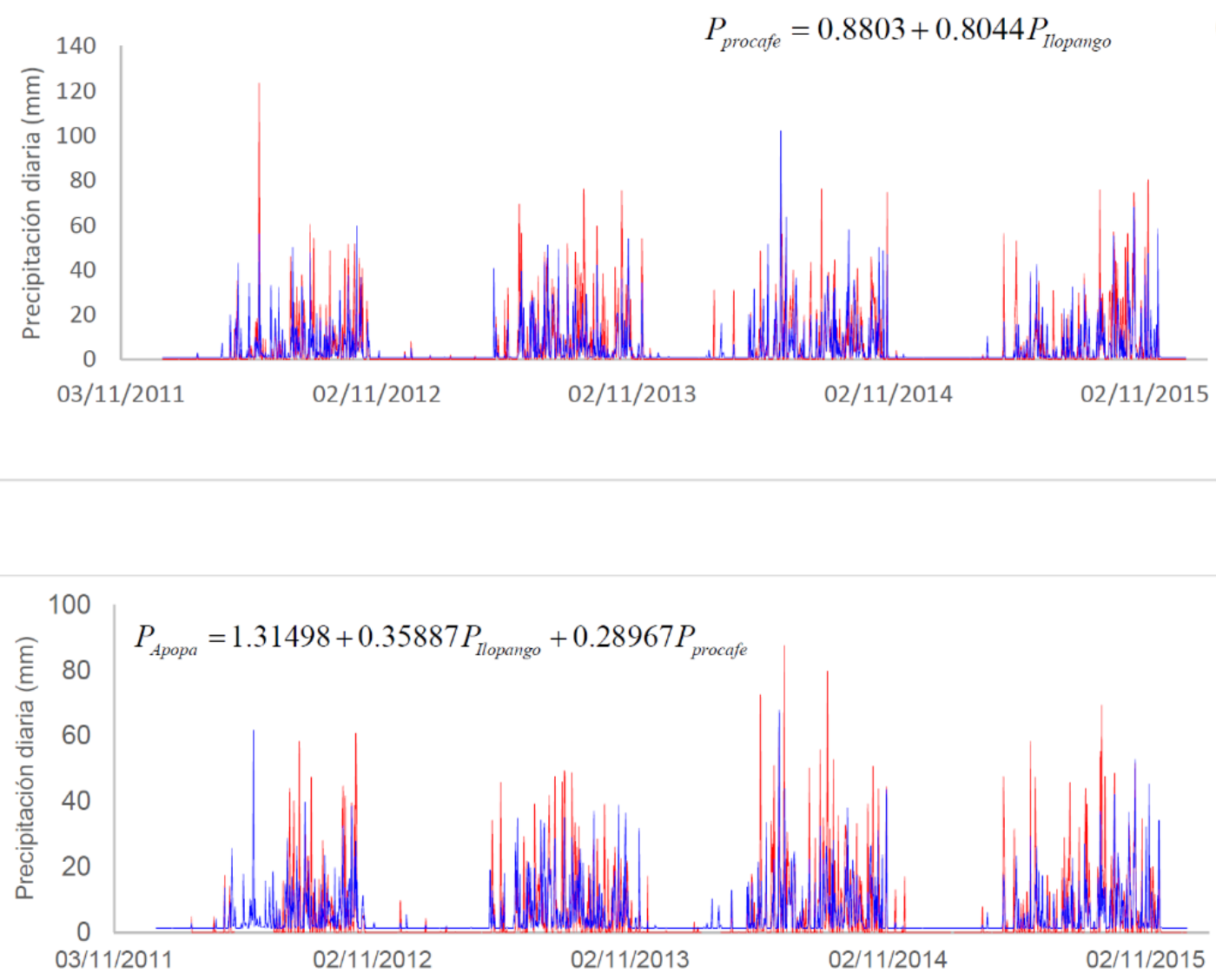

(b)

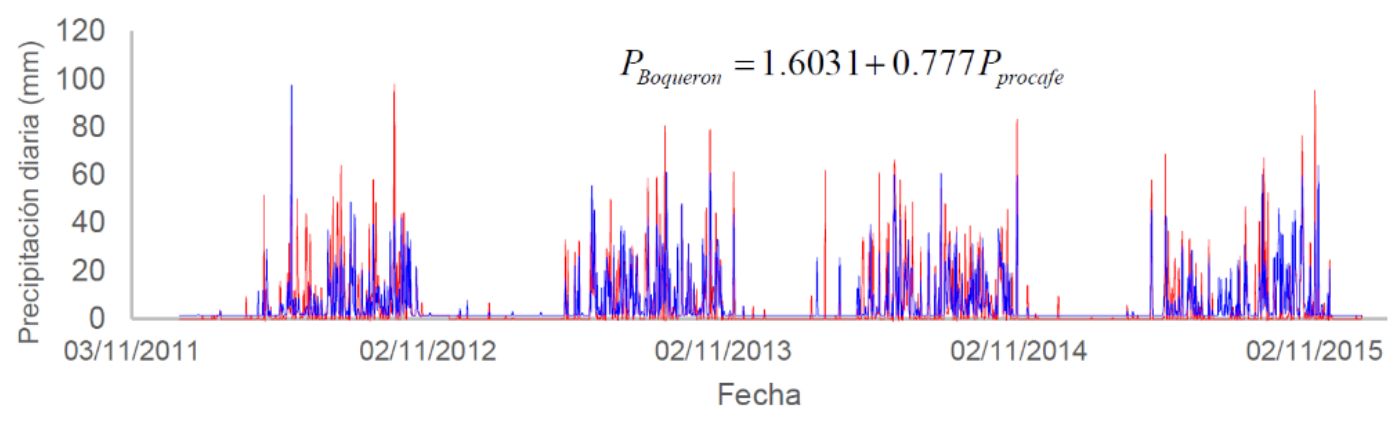

(c)

Valores medidos

Pronóstico 
Figura 2. Comparación de series de datos de precipitación diaria rellenadas para tres estaciones climatológicas de la zona de estudio: a) estación L8 Procafé; b) estación S04 Apopa, y c) estación L18 Boquerón.

A su vez, los datos de temperatura máxima, media y mínima diaria se obtuvieron del registro de monitoreo climático del MARN para las estaciones L18 (1 588 msnm), L8 (964 msnm) y S10 (615 msnm), que cuentan con monitoreo diario de este parámetro entre los años 2005 y 2017. En la estación S17 ( 420 msnm) no se contó con dicho registro, por lo tanto, los valores para la estación S17 se calcularon a partir de la ecuación de la recta que describe la tasa de cambio de temperatura con elevación "lapse rate" (Holden \& Rose, 2011) para la zona. El análisis se hizo a partir de los datos diarios de las tres estaciones para un registro de 12 años comprendido entre 2005 y 2015 (Figura 1 y Figura 3). 
2022, Instituto Mexicano de Tecnología del Agua

Ciencias ¿Agua

Open Access bajo la licencia CCBY-NC-SA 4.0

(https://creativecommons.org/licenses/by-nc-sa/4.0/)

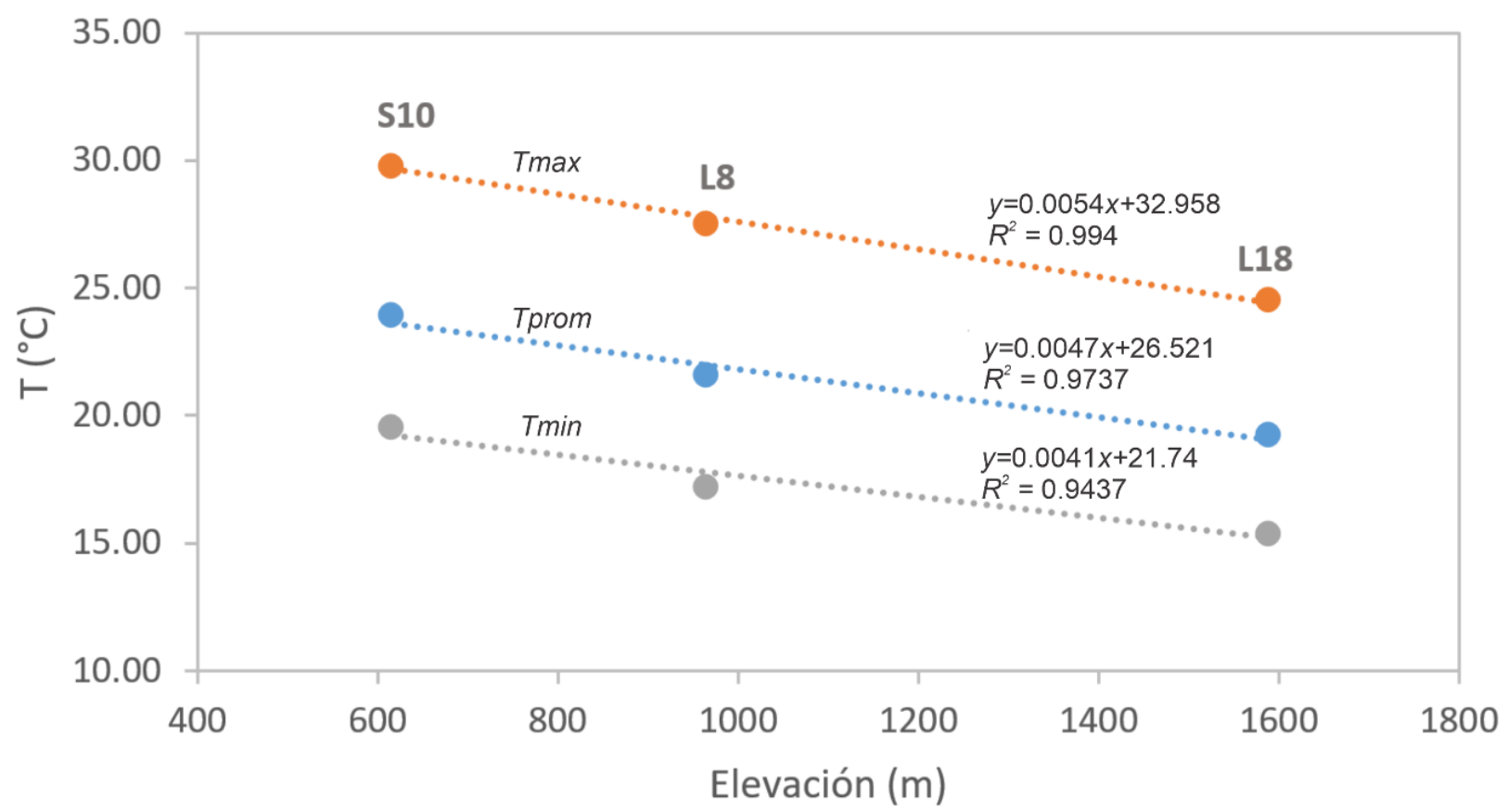

Figura 3. Tasa de cambio de temperatura máxima, media y mínima con elevación en el AMSS para el periodo 2005-2015. Asimismo, se muestra la ecuación de regresión y el valor de $R^{2}$.

El modelo de recarga se elaboró en un dominio de $31.05 \times 29.70$ km y celdas de 90 × 90 m, totalizando 113850 celdas de análisis, que comprendía en su interior a la zona de estudio. Para ello, un total de 1 461 ráster diarios de precipitación, temperatura máxima y mínima se elaboraron para los cuatro años de análisis utilizando el método de la distancia cuadrática inversa mediante un algoritmo programado en GNU Octave (Eaton, Baterman, Hauberg, \& Wehbring, 2019), totalizando 4383 
ráster climáticos para todo el periodo analizado. Por su parte, los ráster de las características físicas y uso del suelo se elaboraron con ArcGis 10.3.

El ráster de usos de suelo (Figura 4a) fue reclasificado y agrupado en 10 usos de los 31 identificados en todo el dominio (ocho usos en la zona de estudio), utilizando el esquema de Anderson Nivel II (Anderson, Hardy, Roach, \& Witmer, 1976) (Figura 4a). El ráster de grupo hidrológico de suelos se elaboró a partir de la textura del suelo detallada en el levantamiento general de suelos (Rico et al., 1965). Las seis texturas identificadas dentro de la zona de estudio también se reclasificaron y agruparon en cuatro grupos hidrológicos de suelo (Figura 4b), atendiendo la clasificación del NRCS y ARS (2003), y tomando en cuenta lo sugerido por Westenbroek et al. (2018). 
Tecnología y

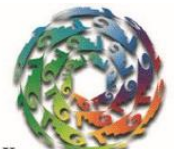

\section{Ciencias}

2022, Instituto Mexicano de Tecnología del Agua

Open Access bajo la licencia CC BY-NC-SA 4.0

(https://creativecommons.org/licenses/by-nc-sa/4.0/)
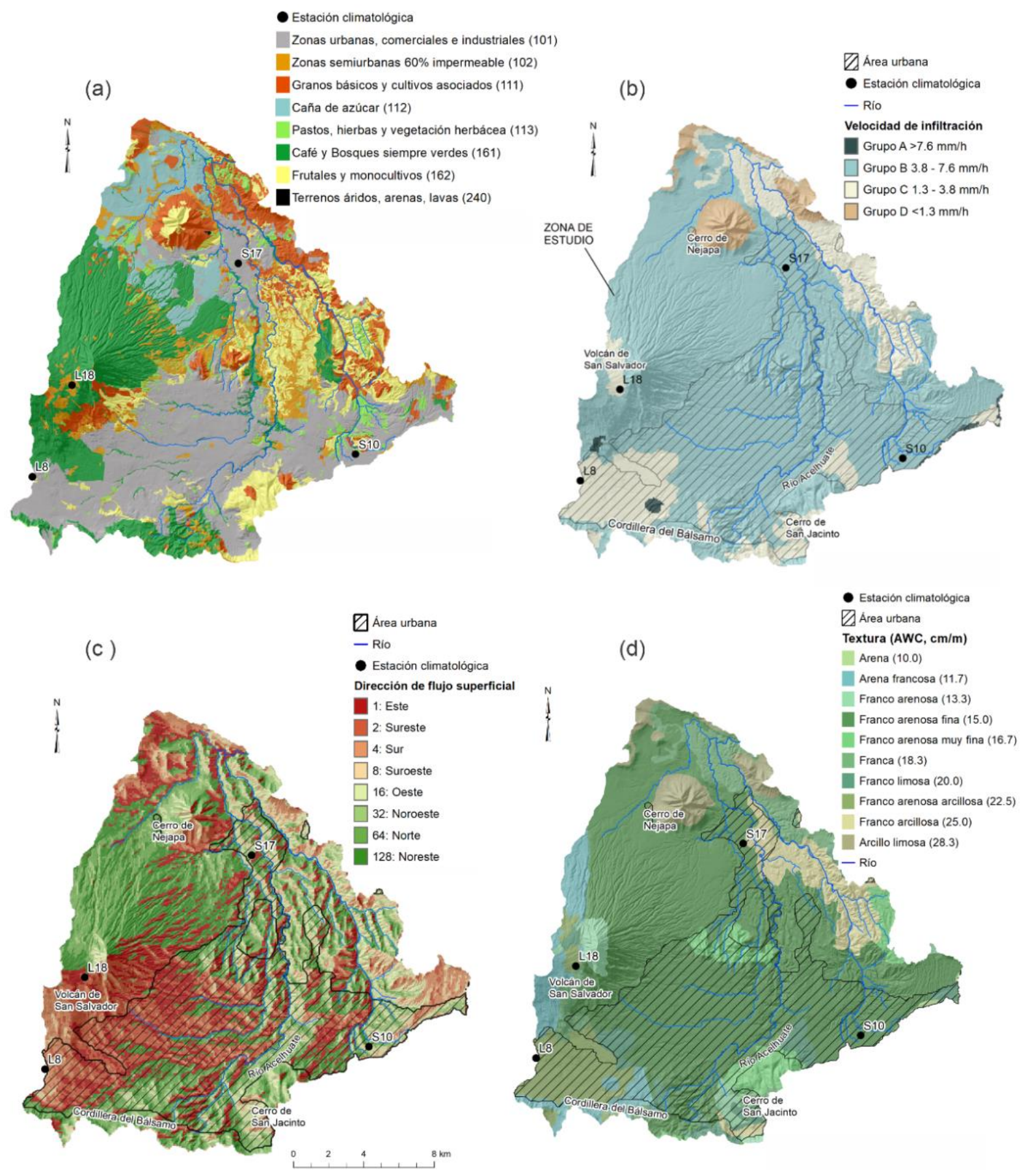
Figura 4. Distribución de las características físicas del AMSS utilizadas en el modelo SWB: a) uso del suelo clasificado en ocho categorías según Anderson Nivel II (Anderson et al., 1976); b) velocidad de infiltración (NRCS \& ARS, 2003), definida a partir de las texturas del suelo según Rico et al. (1965); c) dirección de flujo superficial definido a partir del DEM utilizando el método D8 (Greenlee, 1987); d) capacidad de retención de agua en el suelo.

Por su parte, la malla de dirección de flujo (Figura 4c) se definió a partir del geoprocesamiento del modelo de elevación digital del terreno, utilizando la herramienta Flow Direction en el módulo Hydrology del Spatial Analyst de ArcGis 10.3. Mientras que la malla de la capacidad de agua disponible en el suelo se elaboró a partir de los valores recomendados por Westenbroek, Kelson, Dripps, Hunt y Bradbury (2010) para distintas texturas de suelo (Figura 4d). Dicha variable factorizada con la profundidad de las raíces, definida a partir del mapa de usos de suelo, proporcionó la máxima capacidad de retención de agua en el suelo para cada celda en el dominio del modelo. Este es el valor que debe superar la humedad del suelo en el día de simulación de la Ecuación (1) para contabilizar como recarga. 
A la vez que se preparó las mallas, se configuró un archivo de control para correr el modelo de recarga, en él se indicaron los métodos para cada variable del balance (Ecuación (1)); los archivos de entrada, su formato y proyección espacial; el periodo de simulación, y los archivos de salida. La intercepción se calculó con el método de la cubeta, que asume un porcentaje constante de retención de la Iluvia en el follaje por cada evento de precipitación (Schosinsky, 2006; Siles, Vaast, Dreyer, \& Harmand, 2010).

Por su parte, la escorrentía superficial se calculó mediante el método del número de curva del NRCS, con la variante de la reducción del término de abstracción inicial a un $5 \%$ de la máxima capacidad de almacenamiento de agua en el suelo ( $S_{\max }$ ) (Woodward et al., 2003). Ello implica que la escorrentía inicia con precipitaciones de menor intensidad que con el método original del NRCS.

Para este módulo, los números de curva se asignaron a partir de Cronshey et al. (1986), que se aumentan o disminuyen en el programa a partir de la precipitación de los cinco días previos, pues ello influye sobre las condiciones de humedad antecedente del suelo y la facilidad que tiene para generar escorrentía. Mayores detalles del método pueden consultarse en Westenbroek et al. (2018, 2010). El módulo de aporte de escorrentía de las celdas contiguas "runoff routing" se desactivó para el modelo de recarga, a fin de evitar un sobredimensionamiento del ingreso de agua al suelo. 
Para el cálculo de la evapotranspiración potencial se utilizó el método de Hargreaves-Samani (Hargreaves \& Samani, 1985), apropiado para casos con datos limitados como en la zona de estudio. El método requiere temperaturas máximas y mínimas diarias, así como radiación solar extraterrestre, la cual es calculada en el programa mediante la ubicación espacial de la zona de estudio. Por su parte, la evapotranspiración real se calculó con el método de Thornthwaite-Mather (Thornthwaite, 1948; Thornthwaite \& Mather, 1955; Thornthwaite \& Mather, 1957).

Una vez modelado el sistema, los resultados del SWB se obtuvieron en NetCDF (Network Common Data Form), el cual consiste en un conjunto de librerías y formatos de datos independientes del sistema operativo que admiten la creación, acceso e intercambio de datos científicos orientados a matrices (Rew et al., 1989). Los archivos NetCDF de precipitación, evapotranspiración real, intercepción, escorrentía y recarga fueron procesados utilizando Climate Data Operator (CDO) (Schulzweida, 2019) para obtener los valores anuales de cada variable a partir de la suma de sus valores diarios en cada celda del modelo generando así los ráster de cada variable para los años 2012-2015 en la zona de estudio.

\section{Balance de masa de cloruros (CMB)}


El método de CMB se basa en la propiedad conservativa de los cloruros $\left(\mathrm{Cl}^{-}\right)$y asume que la precipitación es la única fuente de aporte del anión cloruro que ingresa al flujo del agua subterránea (Eriksson \& Khunakasem, 1969). Así, en un evento de precipitación, el flujo del $\mathrm{Cl}^{-}$ depositado en la superficie del suelo es igual al flujo de $\mathrm{Cl}^{-}$que cruza la zona radicular con el agua infiltrada (Flint, Flint, Kwicklis, Fabryka-Martin, \& Bodvarsson, 2002). Cuando el flujo avanza en profundidad, hay agua que se extrae por evapotranspiración, por lo tanto, la concentración de $\mathrm{Cl}^{-}$en el agua de la zona no saturada se incrementa mientras la tasa de infiltración se reduce.

Entonces, la infiltración neta es el flujo de agua que se mueve en la zona fuera del efecto de la evapotranspiración, de forma tal que la concentración de $\mathrm{Cl}^{-}$permanece relativamente constante debajo de esa profundidad y por su carácter conservativo se mueve a la misma velocidad que el agua (Espinosa-Martínez, Custodio, \& Loaso-Vierbücher, 2015). La tasa de infiltración neta se puede estimar a partir de las concentraciones de cloruro utilizando la Ecuación (3):

$R=\frac{P C_{p}}{C_{w g}}$ 
Donde $P$ es la precipitación media anual efectiva por área $(\mathrm{mm}) ; R$, la recarga total $(\mathrm{mm}) ; C_{p}$, la concentración media de cloruro en la precipitación ( $\left.\mathrm{mg} \mathrm{l}^{-1}\right)$, y $C_{w g}$ es la concentración de cloruro en el agua subterránea $\left(\mathrm{mg} \mathrm{l}^{-1}\right)$.

En países como El Salvador, la deposición de $\mathrm{Cl}^{-}$aún no es monitoreada de forma sistemática, por lo tanto, los datos disponibles son escasos y responden a objetivos puntuales. Durante este estudio se monitoreó la concentración de $\mathrm{Cl}^{-}$en lluvia de tres meses: agosto, septiembre y octubre de 2017. Las mediciones se realizaron en dos estaciones localizadas dentro de la zona de estudio. Los resultados de los análisis de $\mathrm{Cl}^{-}$en Iluvia se promediaron con datos reportados por Barrerade-Calderón (2010) en dos manantiales de montaña (Tabla 1 y Figura 1), cuya química se asemeja a la del agua de Iluvia, asumiendo que los manantiales de montaña presentan concentraciones de $\mathrm{Cl}^{-}$similares a los registrados en la precipitación (Flint et al., 2002).

Tabla 1. Concentración de cloruros en Iluvia para cada subzona utilizada en el CMB.

\begin{tabular}{|c|c|c|c|c|c|}
\hline ID & $\boldsymbol{X}(\mathbf{m})$ & $\boldsymbol{Y}(\mathbf{m})$ & $\begin{array}{c}\text { Elevación } \\
\mathbf{( m s n m )}\end{array}$ & Fecha & $\mathbf{C l}^{-}$ \\
\hline \multicolumn{5}{|c|}{ Zong I-1) } \\
\hline $\begin{array}{c}112 \\
\text { (manantial) }\end{array}$ & 469583.46 & 289542.74 & 1728 & $17 / 07 / 2009$ & 4.2 \\
\hline
\end{tabular}


Tecnología y

Ciencias $\stackrel{\Xi}{\Im}$ Aua
2022, Instituto Mexicano de Tecnología del Agua

Open Access bajo la licencia CC BY-NC-SA 4.0

(https://creativecommons.org/licenses/by-nc-sa/4.0/)

\begin{tabular}{|c|c|c|c|c|c|}
\hline $\begin{array}{c}112 \\
\text { (manantial) }\end{array}$ & 469583.46 & 289542.74 & 1728 & $03 / 11 / 2009$ & 2.6 \\
\hline L-18 & 469536.00 & 290480.00 & 1588 & Sept-nov. 2017 & 0.5 \\
\hline L-8 & 468809.00 & 285134.00 & 965 & Sept-nov. 2017 & 0.5 \\
\hline Promedio & \multicolumn{2}{|c|}{ Zona 2 San Jacinto } & & $\mathbf{1 . 9 5}$ \\
\hline $\begin{array}{c}\text { 247 } \\
\text { (manantial) }\end{array}$ & 480697.59 & 282596.44 & 786 & $15 / 07 / 2009$ & 4.2 \\
\hline L-18 & 469536.00 & 290480.00 & 1588 & Sept-nov. 2017 & 0.5 \\
\hline L-8 & 468809.00 & 285134.00 & 965 & Sept-nov. 2017 & 0.5 \\
\hline Promedio & & & & & 1.73 \\
\hline
\end{tabular}

Las concentraciones de $\mathrm{Cl}^{-}$reportados por Barrera-de-Calderón (2010) en los manantiales de montaña oscilan entre 2.60 y $4.2 \mathrm{mg} \mathrm{I}^{-1}$. Estos valores se encuentran en el rango de los valores de $\mathrm{Cl}^{-}$en lluvia reportados para distintas partes del planeta, por ejemplo, Ting, Kerh y Liao (1998) reportaron valores entre 0.90 y $2.19 \mathrm{mg} \mathrm{l}^{-1}$ en Ping Tung, Taiwán. Flint et al. (2002) reportaron una concentración media anual de deposición de $\mathrm{Cl}^{-}$de $0.35 \mathrm{mg} \mathrm{l} \mathrm{I}^{-1}$ en Yucca Mountain, EUA. Por su parte, Lihe, Guangcai, Zhengping y Ying (2010) midieron valores entre 2.30 y $11.79 \mathrm{mg} \mathrm{l}^{-1}$, en Ordos Plateau, China; Naranjo, Cruz-Fuentes, Cabrera, \& Custodio (2015) reportaron valores de 4.2 a $9.2 \mathrm{mg} \mathrm{l}^{-1}$ en Gran Canaria, 
España, y Demlie (2015) registró valores entre 0.37 y $2.02 \mathrm{mg} \mathrm{l}^{-1}$ en Etiopía central.

A partir de la localización de los manantiales con registro de concentraciones de $\mathrm{Cl}^{-}$, la zona de estudio se dividió en dos subzonas (Figura 1), las cuales, a su vez, corresponden con los flujos principales determinados por Barrera-de-Calderón et al. (2021b), donde se establece el modelo hidrogeoquímico conceptual del acuífero de San Salvador. Para cada subzona se determinó la concentración promedio de $\mathrm{Cl}^{-}$(Tabla 1).Las muestras de $\mathrm{Cl}^{-}$en agua subterránea se obtuvieron tomando en cuenta la extensión urbana de la zona de estudio, y la suposición de un ambiente conservativo en el sistema (Scanlon et al., 2002; Seiler \& Gat, 2007; Demlie, 2015; Crosbie, Peeters, Herron, McVicar, \& Herr, 2018). Esto se fundamenta, por una parte, en la ubicación de los puntos de muestreo de agua subterránea, los cuales se localizan dentro del área la zona urbana del AMSS que limita con la parte alta de la cuenca (Figura 1), donde se considera que ocurre la mayor recarga natural que ingresa al acuífero (PNUD, 1972; Gil, 2007; Barrera-de-Calderón 2010; Guevara-Retana, 2013; MARN, 2016a).

Por otra parte, en la selección de las muestras de agua subterránea para el CMB, además del criterio de ubicación, se consideraron sólo las muestras que tuviesen concentraciones del ion nitrato inferiores a $10 \mathrm{mg}$ $I^{-1}$ (Naranjo et al., 2015), y con una relación cloruros:nitratos inferior que 2:1 (BGS, 2004), para descartar la recarga de fuentes distintas a la precipitación, como los sistemas de alcantarillado y cultivos. Asimismo, 
se despreció la influencia geotermal en los puntos analizados debido a que la composición química e isotópica de las muestras de agua subterránea consideradas en el CMB indica que su principal fuente de recarga es la precipitación (Barrera-de-Calderón et al., 2021b) y que la presencia de trazadores característicos de influencia geotermal como el sodio obedece principalmente a procesos de disolución de la roca e intercambio catiónico (Rman, 2016; Neal \& Kirchner, 2000).

Así, para estimar la recarga por medio del CMB se evaluó un conjunto de 11 muestras de agua subterránea de pozos de producción que captan diversos estratos geológicos y manantiales distribuidas en ocho puntos, tomadas durante el año 2009 (Barrera-de-Calderón, 2010); y siete muestras en cinco puntos correspondientes al año 2016 (Figura 1), que forman parte de los análisis químicos realizados por Barrera-deCalderón et al. (2021b). Algunos puntos se repiten en ambos periodos. Además, cuando se reportaron dos muestras para un mismo punto en un mismo año se obtuvo el promedio anual para ese punto.

El balance de masas se calculó para cada muestra a partir de la Iluvia efectiva que ingresa al sistema, esto es, el agua que entra en contacto directamente con el subsuelo e inicia un proceso de descenso por la zona radicular, superándola para luego llegar a la zona saturada. Por ello, la escorrentía e intercepción del follaje de los años precedentes se estimaron como porcentajes medios de la precipitación para la estación Boquerón L18 en la zona 1, e Ilopango S10 en la zona 2 . Ambas variables 
se dedujeron de la precipitación total del año previo para obtener la lluvia efectiva en cada zona (Tabla 2).

Tabla 2. Variables climáticas por zona utilizadas para el cálculo de la precipitación efectiva para cada año de muestreo.

\begin{tabular}{|c|c|c|c|c|c|c|c|c|}
\hline \multirow[b]{2}{*}{ Zona } & \multicolumn{2}{|c|}{ Precipitación (mm) } & \multicolumn{3}{|c|}{ Intercepción (\%) } & \multicolumn{3}{|c|}{ Escorrentía (mm) } \\
\hline & $\begin{array}{c}2009 \\
(\mathrm{~mm})\end{array}$ & $\begin{array}{c}2016 \\
(\mathrm{~mm})\end{array}$ & $\%$ & $\begin{array}{c}2009 \\
(\mathrm{~mm})\end{array}$ & $\begin{array}{c}2016 \\
(\mathrm{~mm})\end{array}$ & $\%$ & $\begin{array}{c}2009 \\
(\mathrm{~mm})\end{array}$ & $\begin{array}{l}2016 \\
(\mathrm{~mm})\end{array}$ \\
\hline 1 & 2090.71 & 1905.20 & 8.19 & 171.23 & 156.04 & 21.93 & 458.49 & 417.81 \\
\hline 2 & 1791.15 & 1877.00 & 9.01 & 161.38 & 169.12 & 18.64 & 333.87 & 349.87 \\
\hline
\end{tabular}

\section{Resultados y discusión}

\section{Variabilidad espacial y temporal de la recarga de agua subterránea simulada mediante el balance de humedad del suelo (SWB)}


La Figura 5 muestra el comportamiento de componentes del balance de humedad del suelo, la media aritmética y desviación estándar en mm año-1 para los años 2012 a 2015. Asimismo, se presenta la cantidad de días de lluvia para cada año modelado. Los resultados indican que la mayor variabilidad de los parámetros es presentada por la precipitación y recarga de agua subterránea (Scanlon et al., 2002; Mair et al., 2013), y en menor medida la evapotranspiración real y escorrentía, mientras que la intercepción y el cambio en el almacenamiento presentaron menos variación. 


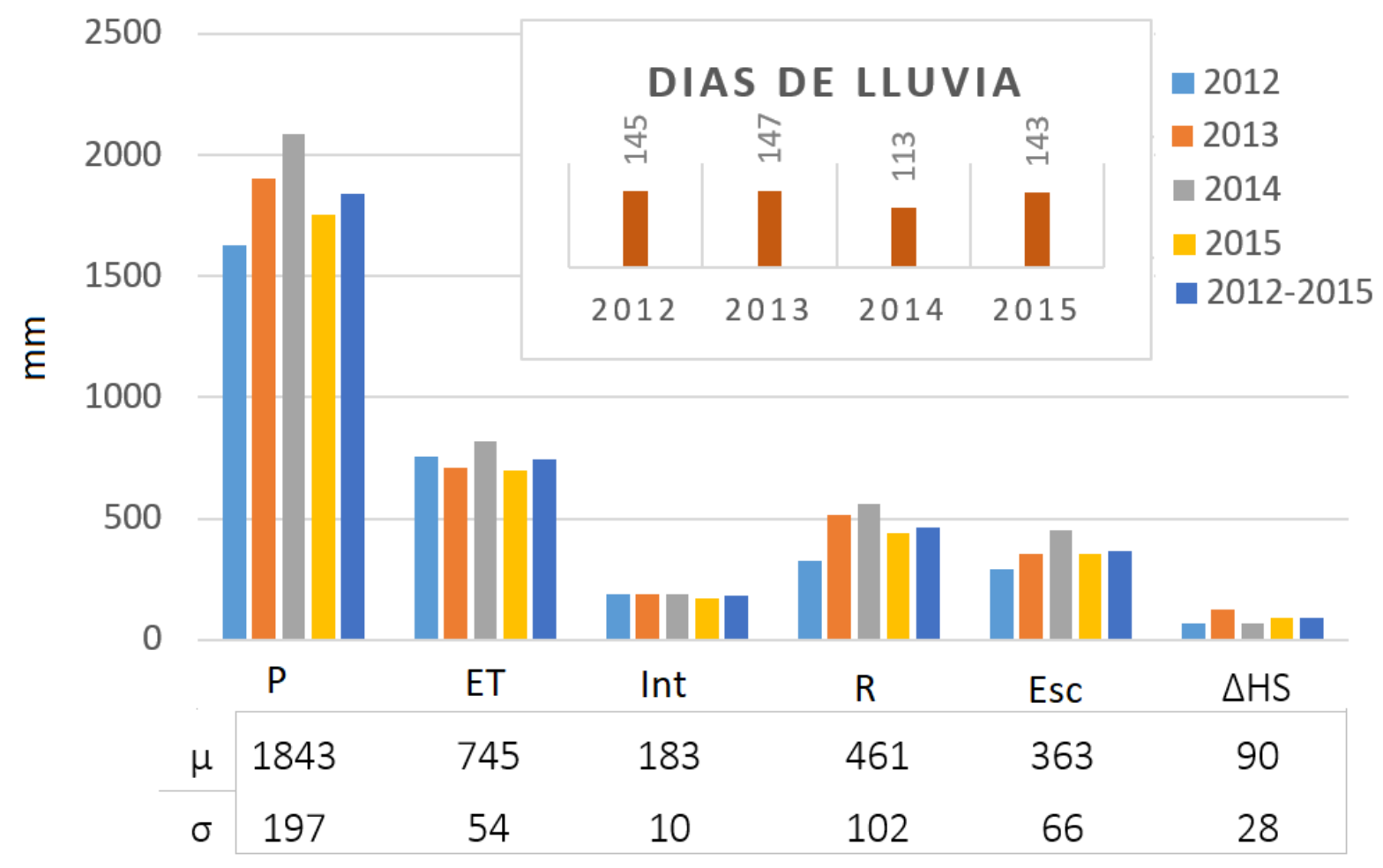

Figura 5. Variación de los componentes del balance hídrico en mm año-

${ }^{1}$ para el acuífero de San Salvador en el periodo 2012-2015 y su promedio multianual. P: precipitación; ET: evapotranspiración real; Int: intercepción; R: recarga; Esc: escorrentía superficial; $\Delta \mathrm{HS}$ : cambio en el almacenamiento.

Para los cuatro años analizados, la recarga varió entre 326 y 561 mm año-1 (20.06-27.04 \% de la lluvia anual), presentando mayores valores para los años más lluviosos (Figura 5). La evapotranspiración resultó con valores entre 698 y 818 mm año-1 (39.7 y $39.18 \%$ ). Por su 
parte, la escorrentía varió entre 291 y $451 \mathrm{~mm}$ año-1 $(17.9-21.6 \%$ de la precipitación), y la intercepción varió entre 168 y $190 \mathrm{~mm}$ año-1, cuyo promedio corresponde a un $9 \%$ de la precipitación total, mientras que el cambio en el almacenamiento reportó los menores valores, que variaron entre 69 y $127 \mathrm{~mm}$ año-1, posiblemente debido a la presencia de la zona urbana, que reduce las áreas permeables disponibles para el ingreso de humedad en el suelo.

Durante el periodo de análisis, el año más lluvioso no corresponde precisamente con el mayor número de días de lluvia, pero sí con la mayor recarga, mostrando una proporcionalidad directa entre la intensidad de la Iluvia y la recarga (p. ej., el año 2014 en la Figura 5), similar a lo reportado por Kendy, Zhang, Liu, Wang y Steenhuis (2004), Crosbie et al. (2012), y Jasechko y Taylor (2015) para climas áridos. El ejemplo más notable es el año 2014 (más lluvioso), en el que resultó el mayor valor de recarga de agua subterránea (Figura 5). Estas lluvias intensas durante el año 2014 fueron reportadas por el MARN (http://mapas.snet.gob.sv/hidrologia) como eventos causantes de inundaciones en algunos puntos dentro de la zona de estudio.

En la Figura 6 se muestran los resultados de la distribución espacial de la recarga de agua subterránea para cada uno de los años estudiados en la zona de estudio. En general, los mayores valores de recarga se presentan en el volcán de San Salvador y cordillera del Bálsamo, coincidiendo con PNUD (1972), Gil (2007), Barrera-de-Calderón (2010), Guevara-Retana (2013) y MARN (2016a). Mientras que la zona urbana 
refleja una notable reducción para todos los años. Los resultados también muestran la importancia de la ladera norte del volcán de San Salvador, no sólo por su significativo aporte a la recarga de agua subterránea, sino porque puede considerarse como una ventana en el tiempo que muestra la evolución de las principales zonas de recarga en la subcuenca del río Acelhuate. 
Tecnología y

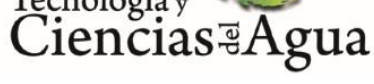

2022, Instituto Mexicano de Tecnología del Agua

Open Access bajo la licencia CC BY-NC-SA 4.0

(https://creativecommons.org/licenses/by-nc-sa/4.0/)
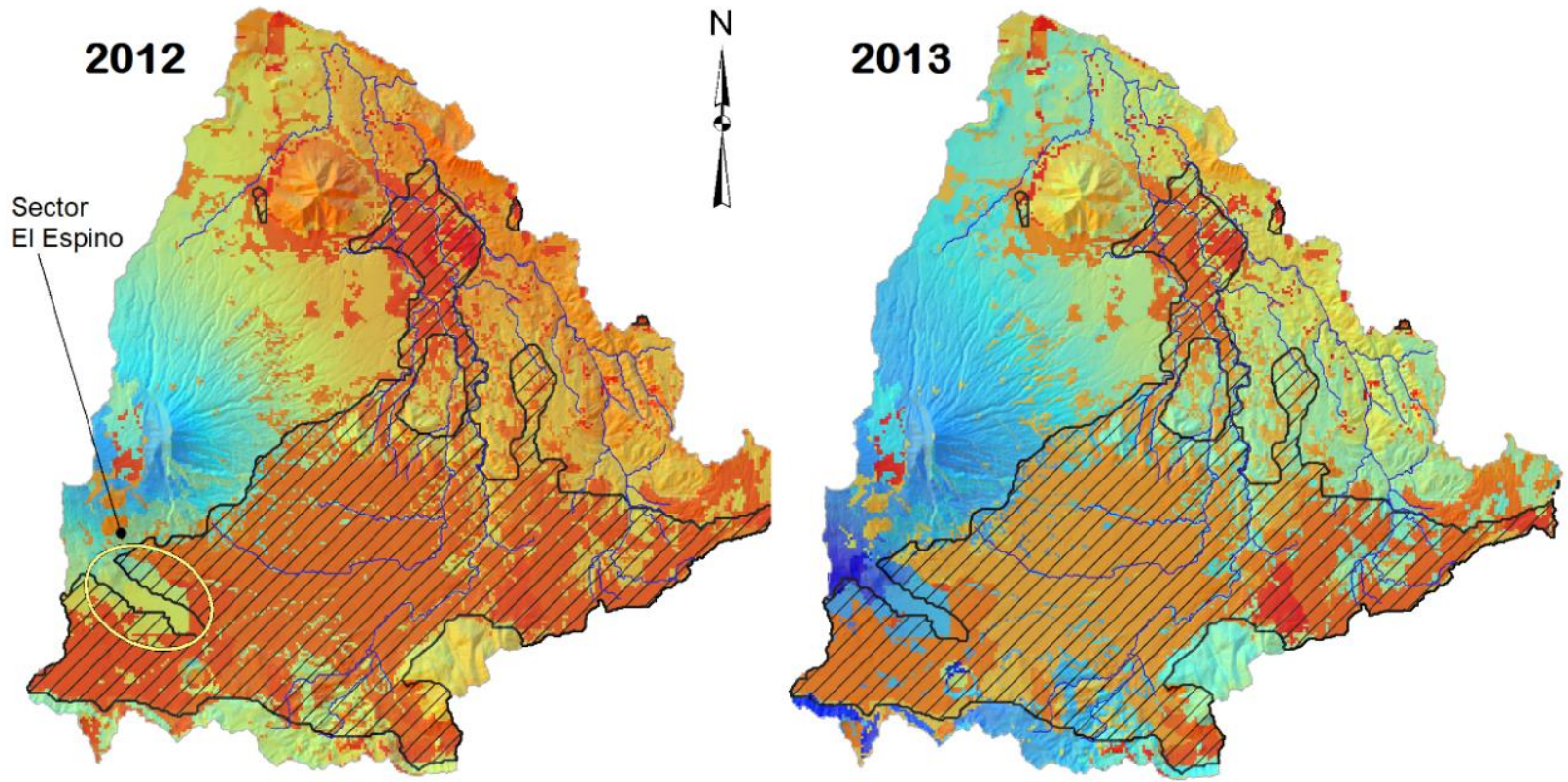

Recarga potencial $(\mathrm{mm})$

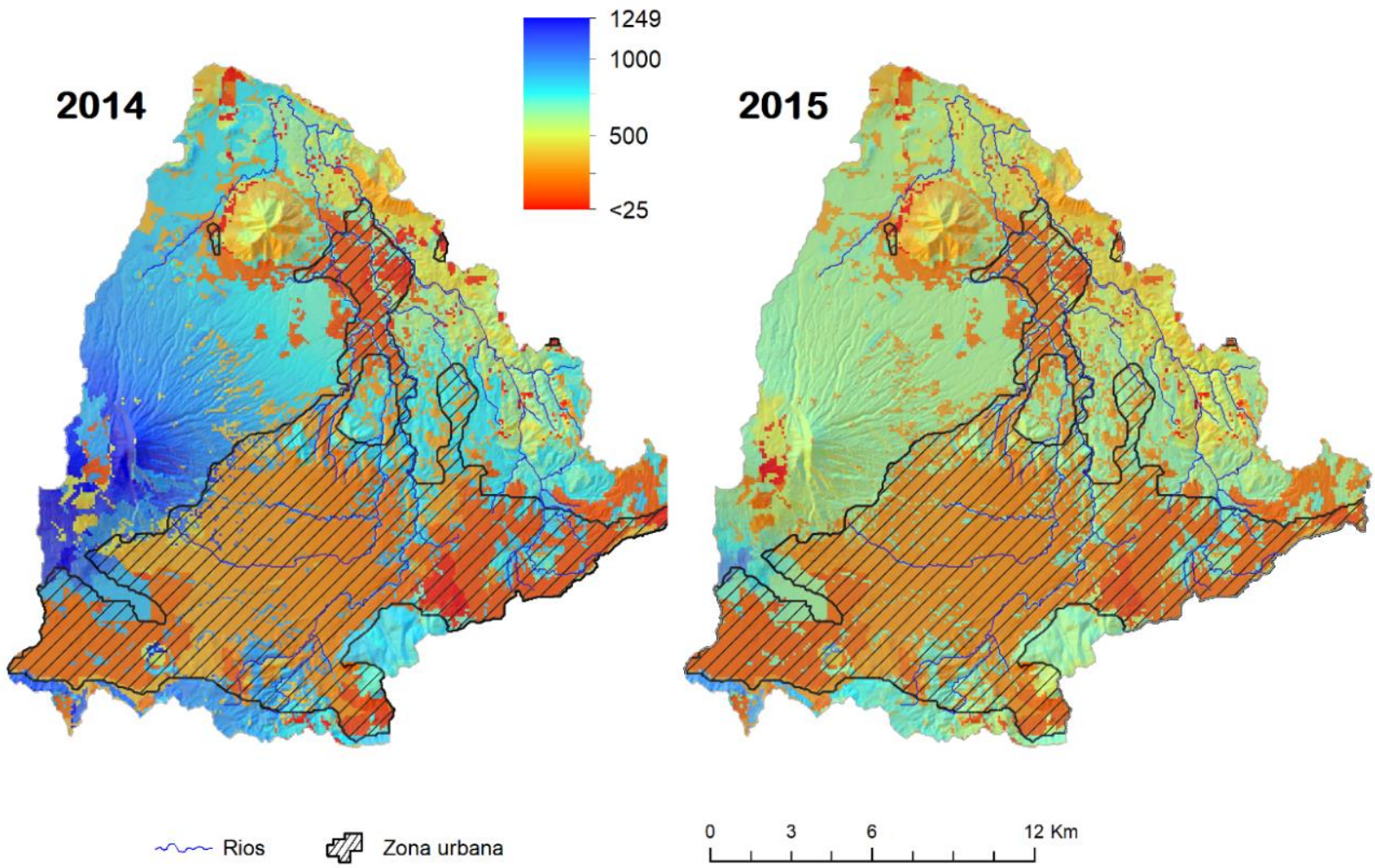

Tecnología y ciencias del agua, ISSN 2007-2422, 13(2), DOI: 10.24850/j-tyca-2022-02-07 
Figura 6. Distribución espacial de la recarga potencial promedio anual de agua subterránea en mm, simulada mediante el SWB para los años 2012 a 2015 en el acuífero urbano de San Salvador, destacando la zona urbana y el sector El Espino en la ladera sureste del volcán de San Salvador.

Los resultados del modelo SWB proveen una idea del impacto que ha ocasionado el avance del desarrollo del AMSS en la recarga de agua subterránea. Las diferencias de recarga entre la ladera norte (sin urbanización), y la ladera oriente y suroriente (con urbanización) en algunos puntos pueden superar los $400 \mathrm{~mm}$ año-1 en años húmedos. Además, los resultados advierten sobre la afectación significativa que puede sufrir el acuífero si se sigue expandiendo el área urbana en las laderas sur y suroriente del volcán de San Salvador sin considerar su importancia hídrica, en particular del sector de la finca El Espino y alrededores que figura como un espacio fuertemente presionado por el desarrollo urbano (MARN, 2017) y con significativa importancia en la sostenibilidad del acuífero para los pozos del AMSS (Barrera-de-Calderón et al., 2021a).

De similar importancia se vuelve la ladera norte del volcán de San Salvador, ya incluida en futuros proyectos urbanísticos del área de estudio y en planes de desarrollo para esa zona. En este caso, los ríos San Antonio y El Ángel, además de importantes manantiales del sector (Barrera-de- 
Calderón et al., 2021 a), podrían mostrar descensos significativos en su caudal base debido a la disminución de la recarga de agua subterránea y, en consecuencia, del nivel potenciométrico en el acuífero.

La elevada importancia del acuífero de San Salvador ha motivado a distintos autores (PNUD, 1972; Servicio Hidrológico Nacional, 2005; MARN, 2016a) a calcular su recarga de agua subterránea por ser un parámetro directamente asociado con la disponibilidad de agua. Todos los estudios anteriores han calculado la recarga de agua subterránea a escala mensual, esta es la primera vez que se realiza un cálculo a nivel diario para un acuífero del país.

La iError! No se encuentra el origen de la referencia. muestra los resultados de tres estudios en los cuales se determinó la recarga de agua subterránea: el PNUD (1972) calculó que la recarga de agua subterránea para cuatro años consecutivos, mediante la separación de hidrogramas, podía variar entre 169 y 221 mm año-1 (10.0 y $11.3 \%$ de la precipitación anual); estos valores son los más bajos reportados en los estudios de la zona y presentan significativas diferencias con estudios posteriores. En su estudio, PNUD (1972) igualó la recarga de agua subterránea a la descarga sin tener en cuenta un flujo subterráneo más profundo, que puede ser significativo (Scanlon et al., 2002).

Tabla 3. Componentes anuales del balance hídrico determinados en estudios realizados en la cuenca alta del río Acelhuate. 
Tecnología y

Ciencias $₫$ Agua
2022, Instituto Mexicano de Tecnología del Agua

Open Access bajo la licencia CC BY-NC-SA 4.0

(https://creativecommons.org/licenses/by-nc-sa/4.0/)

\begin{tabular}{|c|c|c|c|c|c|}
\hline $\mathbf{P}$ & $\mathbf{R}$ & ETR & Esc & $\begin{array}{c}\text { Periodo } \\
\text { de análisis }\end{array}$ & Fuente, método \\
\hline $\begin{array}{c}1673-1 \\
986\end{array}$ & $169-221$ & $\begin{array}{c}1 \text { 171- } 1 \\
390\end{array}$ & $\begin{array}{l}333- \\
375\end{array}$ & 1967-1971 & $\begin{array}{l}\text { PNUD (1972), } \\
\text { separación de } \\
\text { hidrogramas }\end{array}$ \\
\hline 1717.9 & 319 & 881.2 & 517.57 & $1971-2001$ & $\begin{array}{l}\text { Servicio Hidrológico } \\
\text { Nacional (2005), } \\
\text { balance de humedad del } \\
\text { suelo mensual- } \\
\text { multianual }\end{array}$ \\
\hline 1800 & 336 & & 241.9 & $1965-2012$ & $\begin{array}{l}\text { MARN }(2016 a) * \\
\text { balance de humedad del } \\
\text { suelo mensual- } \\
\text { multianual }\end{array}$ \\
\hline $\begin{array}{l}1627- \\
2086\end{array}$ & $326-561$ & $698-818$ & $\begin{array}{l}291- \\
451\end{array}$ & 2012-2015 & $\begin{array}{l}\text { Este estudio**, balance } \\
\text { de humedad del suelo, } \\
\text { diario anual }\end{array}$ \\
\hline
\end{tabular}

*Algunos términos del balance no fueron reportados por los autores.

** No se presenta el valor de intercepción y cambio de almacenamiento. P: precipitación, R: recarga, ETR: evapotranspiración real, Esc.: escorrentía superficial (valor medio).

Los estudios posteriores fueron realizados mediante un balance hídrico de suelos, a nivel mensual con datos multianuales. El Servicio Hidrológico Nacional (2005) determinó que la recarga de agua subterránea en promedio para un periodo de 30 años es de 319 mm año- 
1; por su parte, el MARN (2016a), realizó un análisis similar para un periodo de 48 años, obteniendo una recarga promedio de $336 \mathrm{~mm}$, la más elevada de los estudios comentados en este artículo.

En este estudio, la recarga resultó de 326-561 mm año-1, mayor que en los estudios previos; posiblemente porque los periodos son distintos, se considera la variabilidad temporal y se toma en cuenta el aporte de lluvias intensas (Manna et al., 2019), el cual no se considera cuando se hacen determinaciones a escalas de tiempo menos frecuentes. Otro factor que pudo influir es la diferencia en la distribución de las texturas de suelo, las cuales fueron tomadas en este estudio a una escala de 1:50 000 mientras que en los estudios del Servicio Hidrológico Nacional (2005) y MARN (2016a), la textura se estimó de un mapa a escala 1:100 000. De manera similar pueden compararse otros componentes del balance hídrico. La escorrentía superficial determinada mediante el SWB varía en un rango parecido al determinado por PNUD (1972) y MARN (2016a), e inferior a lo reportado por el Servicio Hidrológico Nacional (2005), mientras que la evapotranspiración real de este estudio resultó menor, lo cual se asocia con el hecho de que estos autores no consideraron la intercepción como uno de sus componentes, dejando más agua disponible en el suelo, los cuales se integran en los cálculos a los procesos de evaporación y transpiración de las plantas (Thornthwaite \& Mather, 1955; Thornthwaite \& Mather, 1957; Allen, 2006). 


\section{Estimación de la recarga de zonas no urbanas mediante el balance de masa de cloruros (CMB)}

La iError! No se encuentra el origen de la referencia. presenta los datos y resultados de valores de recarga de agua subterránea estimados utilizando el método del CMB. Los resultados indican que la recarga varía con la precipitación y concentración de cloruros en el agua subterránea (Berehanu, Azagegn, Ayenew, \& Masetti, 2017). La concentración de cloruros en lluvia resultó de $1.95 \mathrm{mg} \mathrm{l}^{-1}$ para la zona 1 y $1.72 \mathrm{mg} \mathrm{l}^{-1}$ para la zona 2.

Tabla 4. Recarga de agua subterránea en el acuífero de San Salvador determinada mediante el método del CMB para muestras tomadas en los años 2009 y 2016

\begin{tabular}{|c|c|c|c|c|c|c|c|c|c|c|}
\hline ID & Fecha & Zona & $\begin{array}{c}P_{\text {prom }} \\
(\mathbf{m m} \\
\left.\mathbf{a n ̃ o}^{-1}\right)\end{array}$ & $\begin{array}{c}\text { Inter- } \\
\text { cepción } \\
(\mathbf{m m} \\
\left.\mathbf{a n ̃ o}^{-1}\right)\end{array}$ & $\begin{array}{c}\text { Esco- } \\
\text { rrentía } \\
(\mathrm{mm} \\
\text { año-1 }\end{array}$ & $\begin{array}{l}\text { Pefectiva } \\
(\mathbf{m m} \\
\left.\mathbf{a n ̃ o}^{-1}\right)\end{array}$ & $\begin{array}{c}\mathrm{Cl}_{\text {precip }} \\
(\mathrm{mg} \\
\left.\mathrm{L}^{-1}\right)\end{array}$ & $\begin{array}{l}\mathrm{Cl}_{\mathrm{gw}} \\
(\mathrm{mg} \\
\left.\mathrm{L}^{-1}\right)\end{array}$ & $\begin{array}{c}\text { Recarga } \\
(\mathrm{mm} \\
\left.\mathrm{año}^{-1}\right)\end{array}$ & $\begin{array}{c}\text { Recarga } \\
\text { promedio } \\
(\mathbf{m m} \\
\text { año-1 }\end{array}$ \\
\hline \multicolumn{11}{|c|}{ AÑO 2009} \\
\hline 2 & $23 / 06 / 2009$ & 1 & 2090.71 & 171.23 & 458.49 & 1460.99 & 1.95 & 8.51 & 334.77 & 444.52 \\
\hline 2 & 29/10/2009 & 1 & 2090.71 & 171.23 & 458.49 & 1460.99 & 1.95 & 5.14 & 554.27 & \\
\hline
\end{tabular}


Tecnología y

Ciencias $\approx$ Agua
2022, Instituto Mexicano de Tecnología del Agua

Open Access bajo la licencia CC BY-NC-SA 4.0

(https://creativecommons.org/licenses/by-nc-sa/4.0/)

\begin{tabular}{|c|c|c|c|c|c|c|c|c|c|c|}
\hline 3 & 08/09/2009 & 1 & 2090.71 & 171.23 & 458.49 & 1460.99 & 1.95 & 9.27 & 307.33 & 343.59 \\
\hline 3 & 24/11/2009 & 1 & 2090.71 & 171.23 & 458.49 & 1460.99 & 1.95 & 7.50 & 379.86 & \\
\hline 18 & 08/09/2009 & 1 & 2090.71 & 171.23 & 458.49 & 1460.99 & 1.95 & 6.31 & 451.49 & 451.49 \\
\hline 28 & 03/09/2009 & 2 & 1791.15 & 161.38 & 333.87 & 1310.58 & 1.73 & 4.44 & 510.66 & 510.66 \\
\hline 68 & 28/10/2009 & 1 & 2090.71 & 171.23 & 458.49 & 1460.99 & 1.95 & 4.11 & 693.17 & 693.17 \\
\hline 80 & 03/09/2009 & 2 & 1791.15 & 161.38 & 333.87 & 1310.58 & 1.73 & 7.40 & 306.39 & 589.22 \\
\hline 80 & 04/11/2009 & 2 & 1791.15 & 161.38 & 333.87 & 1310.58 & 1.73 & 2.60 & 872.04 & \\
\hline 105 & 24/11/2009 & 1 & 2090.71 & 171.23 & 458.49 & 1460.99 & 1.95 & 9.10 & 313.07 & 313.07 \\
\hline 111 & $15 / 07 / 2009$ & 2 & 1791.15 & 161.38 & 333.87 & 1310.58 & 1.73 & 6.30 & 359.89 & 359.89 \\
\hline \multicolumn{10}{|c|}{ Recarga promedio } & 463.20 \\
\hline \multicolumn{10}{|r|}{ Máximo } & 693.17 \\
\hline \multicolumn{10}{|r|}{ Mínimo } & 313.07 \\
\hline \multicolumn{11}{|c|}{ AÑO 2016} \\
\hline 2 & $29 / 11 / 2016$ & 1 & 1905.20 & 156.04 & 417.81 & 1331.35 & 1.95 & 3.47 & 748.17 & 748.17 \\
\hline 7 & $11 / 11 / 2016$ & 2 & 1877.00 & 169.12 & 349.87 & 1373.40 & 1.73 & 2.98 & 797.31 & 797.31 \\
\hline 31 & $10 / 11 / 2016$ & 1 & 1905.20 & 156.04 & 417.81 & 1331.35 & 1.95 & 4.96 & 523.42 & 523.42 \\
\hline 80 & $01 / 12 / 2016$ & 2 & 1877.00 & 169.12 & 349.87 & 1373.40 & 1.73 & 6.45 & 368.37 & 368.37 \\
\hline 95 & $10 / 11 / 2016$ & 1 & 1905.20 & 156.04 & 417.81 & 1331.35 & 1.95 & 2.98 & 871.19 & 871.19 \\
\hline 105 & $14 / 11 / 2016$ & 1 & 1905.20 & 156.04 & 417.81 & 1331.35 & 1.95 & 6.95 & 373.55 & 373.55 \\
\hline 106 & $14 / 11 / 2016$ & 1 & 1905.20 & 156.04 & 417.81 & 1331.35 & 1.95 & 6.95 & 373.55 & 373.55 \\
\hline \multicolumn{10}{|c|}{ Recarga promedio } & 579.36 \\
\hline \multicolumn{10}{|r|}{ Máximo } & 871.19 \\
\hline \multicolumn{10}{|r|}{ Mínimo } & 368.37 \\
\hline
\end{tabular}

Clprecip: concentración de cloruros en precipitación, $\mathrm{Cl}_{\mathrm{gw}}$ : concentración de cloruros en agua subterránea.

Tecnología y ciencias del agua, ISSN 2007-2422,13(2), DOI: 10.24850/j-tyca-2022-02-07 
Por su parte, las muestras de agua subterránea registraron valores entre 2.6 y $9.27 \mathrm{mg} \mathrm{l}^{-1} \mathrm{de} \mathrm{Cl}^{-}$(Tabla 4); este aumento de la concentración de $\mathrm{Cl}^{-}$en las muestras analizadas es un claro indicador de la pérdida de agua en los procesos de evapotranspiración (Ting et al., 1998; Flint et al., 2002; Lihe et al., 2010). Para los dos periodos evaluados, la concentración de $\mathrm{Cl}^{-}$en el agua subterránea se incrementó entre 1.5 y 4.8 veces la concentración de entrada.

Los valores de recarga para las muestras del año 2009 resultaron entre 313 y 693 mm año-1; mientras que para las muestras de agua subterránea tomadas en el año 2016, el CMB indicó recarga en el rango de 368 y $871 \mathrm{~mm}$ año-1 (Figura 7). Los mayores valores de recarga para ambos periodos se presentan en el sector sur de la cuenca (511-871 mm año-1), en los pozos localizados en las faldas del volcán de San Salvador y cerro de San Jacinto. Mientras que en los pozos del norte, la recarga varía entre 313 y $451 \mathrm{~mm}$ año-1. En las faldas del cerro de San Jacinto, la recarga oscila entre 360 y $589 \mathrm{~mm}^{2}$ ño-1. 
Tecnología y

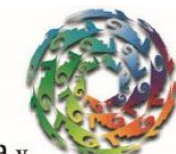

2022, Instituto Mexicano de Tecnología del Agua

Open Access bajo la licencia CC BY-NC-SA 4.0

(https://creativecommons.org/licenses/by-nc-sa/4.0/)

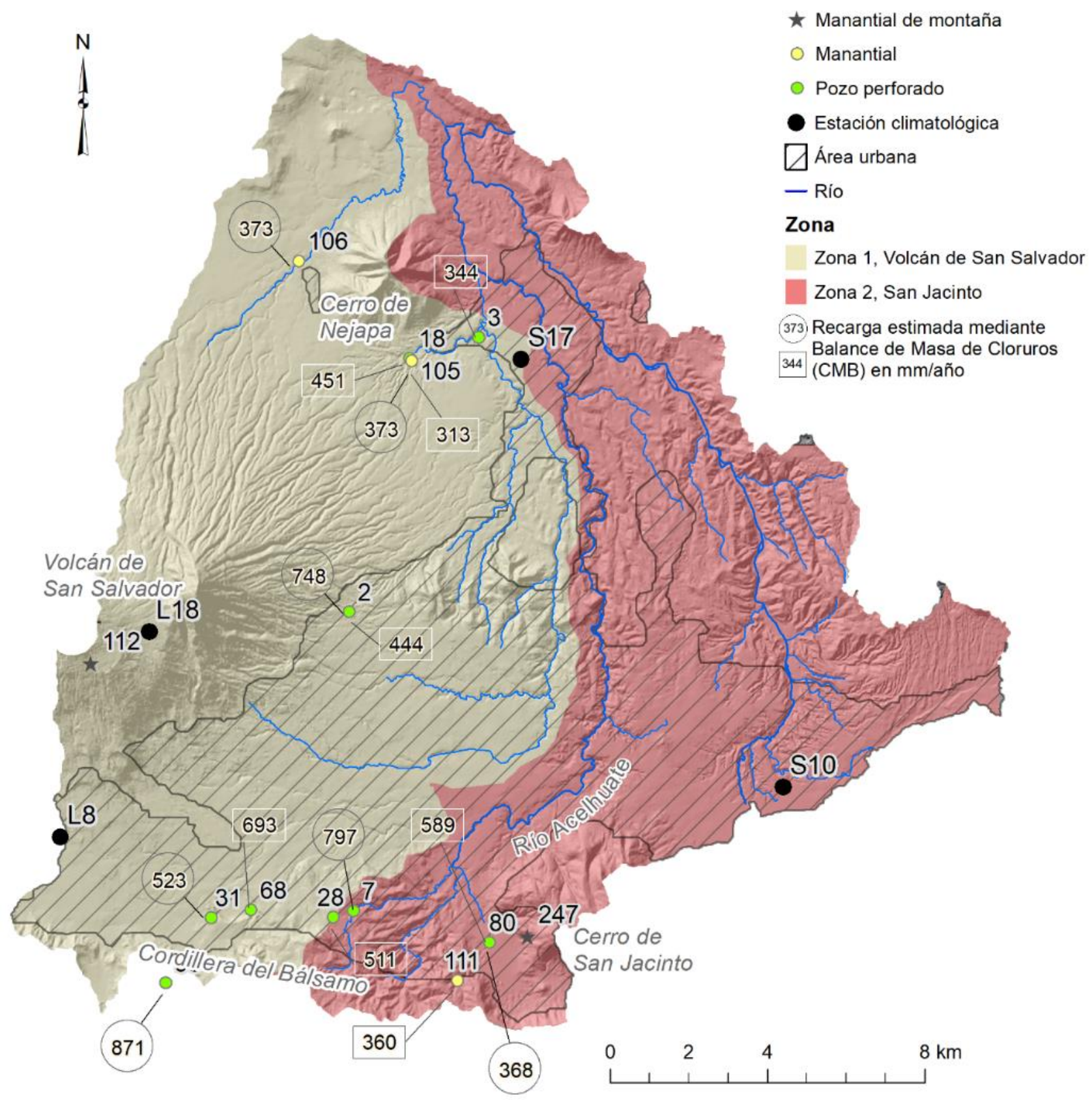

Tecnología y ciencias del agua, ISSN 2007-2422, 13(2), DOI: 10.24850/j-tyca-2022-02-07 
Figura 7. Recarga de la zona no urbana estimada mediante el CMB para el acuífero de San Salvador en muestras tomadas durante los años 2009 (rectángulos) y 2016 (círculos). Los valores asociados con línea al pozo o manantial indican la recarga en $\mathrm{mm}$ año-1.

Aunque los puntos analizados en cada periodo no son los mismos, en general los datos indican que la recarga de las zonas no urbanizadas en la parte alta de la cuenca podría estar variando entre 463 y 579 mm año-1 , 22.8 y $30.6 \%$ de la precipitación, respectivamente; mostrando consistencia con los resultados del SWB y generando mayor confianza en los datos obtenidos en este estudio.

Los resultados de recarga de agua subterránea obtenidos mediante el CMB en una región tropical son consistentes con otros estudios realizados utilizando este método en zonas similares. Demlie (2015) estimó la recarga de agua subterránea para el centro de Etiopía, obteniendo un $25 \%$ de la precipitación media anual. Y aunque el método es principalmente aplicado en regiones con clima árido y semiárido como lo indica una completa recopilación de estudios a nivel global realizado por Scanlon et al. (2006), los resultados parecen aceptables si se tiene el cuidado de cuantificar las variables que implican la estimación de la precipitación efectiva y la viabilidad de desestimar otras fuentes de ingreso de cloruros al sistema. 


\section{Conclusiones}

Los dos métodos utilizados para estimar la recarga directa por precipitación en la subcuenca alta del río Acelhuate (SWB 2.0 y CMB) indican que existe una fuerte variabilidad espacial y temporal de este aporte al acuífero. Mediante el uso del modelo SWB 2.0 fue posible evidenciar que la recarga media anual de la zona en un año seco puede ser de $326 \mathrm{~mm}$ año-1, mientras que en un año húmedo este valor puede alcanzar $561 \mathrm{~mm}$ año-1. Por su parte, el CMB, que fue analizado para dos años con características climáticas húmedas similares, indica que estos valores para la zona alta de la cuenca pueden oscilar entre 313 y $693 \mathrm{~mm}$ año-1, mostrando consistencia en los resultados del SWB. Para ambos métodos, la recarga de agua subterránea representa porcentajes con respecto a la precipitación anual, que varían entre 20 y $30 \%$.

La intensidad y frecuencia de la lluvia son factores fundamentales para el proceso de recarga en esta subcuenca con clima húmedo. Mediante el modelo de lluvia diaria del SWB 2.0 fue posible visualizar que se requiere de una serie de eventos intensos y consecutivos de precipitación para que la humedad supere la zona radicular y pueda cuantificarse como recarga de agua subterránea. 
Por su parte, la utilidad del CMB para determinar la recarga de agua subterránea en una cuenca urbana con clima húmedo ha sido demostrada en este estudio. Tres aspectos relevantes deben destacarse a partir de los resultados obtenidos: primero, el CMB es de bajo costo, versátil y de fácil aplicación, ya que puede utilizarse para estimar la recarga en las áreas sin un evidente impacto urbano, brindando importante información sobre los aportes de las zonas de recarga que suplen a los pozos aprovechados aguas abajo en el acuífero; segundo, el uso de este método requiere la estimación precisa de la precipitación efectiva, pues la recarga está directamente relacionada con esta variable, ello destaca la necesidad de un monitoreo hidrológico apropiado o el uso de métodos hidrológicos alternativos, tal y como fue realizado en este estudio; tercero, debe hacerse un cuidadoso análisis para la selección de los puntos de muestreo, un estricto seguimiento de los protocolos de muestreo y análisis, y la depuración de los resultados químicos a utilizar en el cálculo.

El aumento de la escala temporal en la estimación de la recarga de agua subterránea muestra la importancia de incorporar este tipo de métodos de estimación de los recursos para los procesos de planificación hídrica. La fuerte variabilidad temporal de la recarga deja claridad sobre una diferencia significativa en la estimación de los recursos que debe ser considerada. En la subcuenca alta del río Acelhuate, las estimaciones previas de recarga media a escala mensual con datos climáticos multianuales están cercanos al límite inferior determinado mediante el modelo diario. Sin embargo, no debe perderse de vista que el balance a 
nivel de cuenca no considera precisamente la recarga de las zonas de explotación más intensa, que para este acuífero se localizan en la parte alta, donde las zonas de recarga han experimentado una fuerte reducción debido a la expansión del ambiente urbano.

Además, la variabilidad espacial de la recarga de agua subterránea es también de suma importancia, sobre todo para los procesos de ordenamiento territorial donde se deben definir y proteger las áreas de mayor recarga, de las cuales depende principalmente la renovación anual de agua disponible en el acuífero para los distintos usuarios en la subcuenca, además de la descarga a importantes manantiales que representan el caudal base de ríos como San Antonio, El Ángel y otros que son aprovechados en su totalidad para el abastecimiento de la población del AMSS.

\section{Agradecimientos}

Este trabajo fue financiado mediante una beca otorgada por la Organización de Estados Americanos (OEA). Los autores desean agradecer especialmente el apoyo recibido por el M.Sc. Stephen M. Westenbroek, hidrólogo del USGS en la División de Recursos Hídricos, Centro de Recursos del Agua de Wisconsin, por haber atendido nuestras consultas sobre el modelo SWB 2.0. Agradecemos, además, el valioso apoyo de la Administración Nacional de Acueductos y Alcantarillados (ANDA), Ministerio de Medio Ambiente y Recursos Naturales de El 
Salvador, Universidad Autónoma del Estado de México, Universidad de El Salvador, Conacyt y Comecyt de México, e Institut National de la Recherche Scientifique, Centre Eau, Terre et Environnement (INRS-ETE).

\section{Referencias}

Allen, R. G. (2006). Evapotranspiración del cultivo: guías para la determinación de los requerimientos de agua de los cultivos. Roma, Italia: Organización de las Naciones Unidas para la Agricultura y la Alimentación.

ANDA, Administración Nacional de Acueductos y Alcantarillados. (2008). Mapa Hidrogeológico de la República de El Salvador. EsC. 1:100,000. San Salvador, El Salvador: Administración Nacional de Acueductos y Alcantarillados.

Anderson, J. R., Hardy, E. E., Roach, J. T., \& Witmer, R. E. (1976). A land use and land cover classification system for use with remote sensor data (Geological Survey Professional Paper No. 964). Washington DC, USA: U.S. Geological Surge.

Barrera-de-Calderón, M. (2010). Caracterización hidrogeoquímica e isotópica de áreas de recarga en el acuífero de San Salvador (tesis de maestría). Universidad de El Salvador, El Salvador.

Barrera-de-Calderón, M., Garfias, J., Martel, R., \& Salas-García, J. (2021a). Controles geológicos en el flujo de agua subterránea y distribución de volúmenes de extracción asociados a una estructura 
volcánica compleja. Boletín de la Sociedad Geológica Mexicana 73(1). Recuperado de http://dx.doi.org/10.18268/BSGM2021v73n1a170820.

Barrera-de-Calderón, M., Garfias, J., Martel, R., \& Salas-García, J. (2021b). Impacto urbano en la calidad y recarga del agua subterránea utilizando trazadores hidrogeoquímicos y ambientales en el acuífero de San Salvador. Tecnología y Ciencias del Agua 12(5), 1-52. DOI: https://doi.org/10.24850/j-tyca-2021-05-01

BGS, British Geological Survey. (2004). Water quality fact sheet: Nitrate. United Kingdom: British Geological Survey. Recuperado de http://nora.nerc.ac.uk/id/eprint/516304/1/Nitrate.pdf

Berehanu, B., Azagegn, T., Ayenew, T., \& Masetti, M. (2017). Inter-basin groundwater transfer and multiple approach recharge estimation of the upper awash Aquifer System. Journal of Geoscience and Environment Protection 5(3), 76-98. Recuperado de http://dx.doi.org/10.4236/gep.2017.53007

Cao, G., Scanlon, B. R., Han, D., \& Zheng, C. (2016). Impacts of thickening unsaturated zone on groundwater recharge in the North China Plain. Journal of Hydrology, 537, 260-270. Recuperado de https://doi.org/10.1016/j.jhydrol.2016.03.049

Carr, M. J., \& Stoiber, R. E. (1977). Geologic setting of some destructive earthquakes in Central America. Geological Society of America Bulletin, 88(1), 151-156. Recuperado de 
https://doi.org/10.1130/0016-

7606(1977)88<151:GSOSDE > 2.0.CO;2

Cronshey, R., McCuen, R. H., Miller, N., Rawls, W., Robbins, S., \& Woodward, D. E. (1986). Urban hydrology for small watersheds Technical Release 55 (Draft). Washington DC, USA: U.S. Department of Agriculture, Natural Resource Conservation Service, Conservation Engineering Division.

Crosbie, R. S., Peeters, L. J. M., Herron, N., McVicar, T. R., \& Herr, A. (2018). Estimating groundwater recharge and its associated uncertainty: Use of regression kriging and the chloride mass balance method. Journal of Hydrology, 561, 1063-1080. Recuperado de https://doi.org/10.1016/j.jhydrol.2017.08.003

Crosbie, R. S., McCallum, J. L., Walker, G. R., \& Chiew, F. H. S. (2012). Episodic recharge and climate change in the Murray-Darling Basin, Australia. Hydrogeology Journal, 20(2), 245-261. Recuperado de https://doi.org/10.1007/s10040-011-0804-4

Davis, K. W., \& Putnam, L. D. (2013). Conceptual and numerical models of groundwater flow in the Ogallala aquifer in Gregory and Tripp Counties, South Dakota, water years 1985-2009. (U.S. Geological Survey Scientific Investigations No. Report 2013-5069). Reston, USA: U.S. Geological Survey.

Day, E., \& Simpkins, W. W. (2018). Application of the USGS Soil-WaterBalance (SWB) Model to estimate groundwater recharge in Central 
Iowa. $52^{\circ}$ Annual North-Central Geological Society of America Section Meeting-2018. Recuperado de https://doi.org/10.1130/abs/2018NC-313243

Demlie, M. (2015). Assessment and estimation of groundwater recharge for a catchment located in highland tropical climate in central Ethiopia using catchment soil-water balance (SWB) and chloride mass balance (CMB) techniques. Environmental Earth Sciences, 74(2), 1137-1150. Recuperado de https://doi.org/10.1007/s12665-015-4099-y

Digestyc, Dirección General de Estadística y Censos. (2008). VI Censo de Población y V de vivienda 2007 (Tomo IV), Cifras Oficiales Población-Vivienda-Hogar. San Salvador, El Salvador: Ministerio de Economía, Dirección General de Estadística y Censos.

Doble, R. C., \& Crosbie, R. S. (2017). Review: Current and emerging methods for catchment-scale modelling of recharge and evapotranspiration from shallow groundwater. Hydrogeology Journal, 25(1), 3-23. Recuperado de https://doi.org/10.1007/s10040-016-1470-3

Dripps, W. R., \& Bradbury, K. R. (2009). The spatial and temporal variability of groundwater recharge in a forested basin in northern Wisconsin. Hydrological Processes, 24(4), 383-392. Recuperado de https://doi.org/10.1002/hyp.7497 
Dripps, W. R., \& Bradbury, K. R. (2007). A simple daily soil-water balance model for estimating the spatial and temporal distribution of groundwater recharge in temperate humid areas. Hydrogeology Journal, 15(3), 433-444. Recuperado de https://doi.org/10.1007/s10040-007-0160-6

Eaton, J.W., Baterman, D., Hauberg, S., \& Wehbring, R. (2019). GNU Octave version 5.1.0 manual: a high-level interactive language for numerical computations [software].

Edmunds, W. M., \& Gaye, C. B. (1994). Estimating the spatial variability of groundwater recharge in the Sahel using chloride. Journal of Hydrology, 156(1), 47-59. Recuperado de https://doi.org/10.1016/0022-1694(94)90070-1

Eriksson, E., \& Khunakasem, V. (1969). Chloride concentration in groundwater, recharge rate and rate of deposition of chloride in the Israel Coastal Plain. Journal of Hydrology, 7(2), 178-197. Recuperado de https://doi.org/10.1016/0022-1694(69)90055-9

Espinosa-Martínez, S., Custodio, E., \& Loaso-Vierbücher, C. (2015). Comparación de la recarga natural estimada con el balance de agua en el suelo y con el balance de la deposición atmosférica de cloruro en un sistema carbonatado en el área semiárida del Baix Ebre, Cataluña, España. Ingeniería del Agua 19(3), 135-155. Recuperado de https://doi.org/10.4995/ia.2015.3540 
Flint, A. L., Flint, L. E., Kwicklis, E. M., Fabryka-Martin, J. T., \& Bodvarsson, G. S. (2002). Estimating recharge at Yucca Mountain, Nevada, USA: comparison of methods. Hydrogeology Journal, 10(1), 180-204. Recuperado de https://doi.org/10.1007/s10040001-0169-1

Gil, L. B. (2007). Evaluación hidrogeológica y vulnerabilidad intrínseca del sistema acuífero del municipio de Nejapa, San Salvador, El Salvador (tesis de maestría). Universidad de Costa Rica, Costa Rica.

Greenlee, D. D. (1987). Raster and vector processing for scanned linework. Photogrammetric Engineering and Remote Sensing, 53(10), 1383-1387. Recuperado de https://doi.org/0099$1112 / 87 / 5310-1383 \$ 02.25 / 0$

Guevara-Retana, J. M. (2013). Estudio hidrogeológico área del ingenio azucarero El Angel, jurisdicción de Apopa, departamento de San Salvador (Informe Técnico) (no publicado). San Salvador, El Salvador.

Hargreaves, G. H., \& Samani, Z. A. (1985). Reference crop evapotranspiration from ambient air temperature. 1985 Winter Meeting American Society of Agricultural Engineers, Chicago, IL, 1(2). Recuperado de https://doi.org/10.13031/2013.26773

Healy, R. W., \& Cook, P. G. (2002). Using groundwater levels to estimate recharge. Hydrogeology Journal, 10(1), 91-109. Recuperado de https://doi.org/10.1007/s10040-001-0178-0 
Healy, R. W., \& Scanlon, B. R. (2010). Estimating groundwater recharge. New York, USA. Cambridge University Press.

Heppner, C. S., Nimmo, J. R., Folmar, G. J., Gburek, W. J., \& Risser, D. W. (2007). Multiple-methods investigation of recharge at a humidregion fractured rock site, Pennsylvania, USA. Hydrogeology Journal, 15(5), 915-927. Recuperado de https://doi.org/10.1007/s10040-006-0149-6

Hernández, W., \& Jicha, B. (2019). Múltiple colapso sectorial del volcán de San Salvador, El Salvador, América Central. Revista de la Universidad Tecnológica de El Salvador. Recuperado de URI http://hdl.handle.net/11298/1105

Holden, J., \& Rose, R. (2011). Temperature and surface lapse rate change: a study of the UK's longest upland instrumental record. International Journal of Climatology, 31, 907-919. Recuperado de https://doi.org/10.1002/joc. 2136

Ifediegwu, I. S. (2020). Groundwater recharge estimation using chloride mass balance: A case study of Nsukka local government area of Enugu State, Southeastern, Nigeria. Modeling Earth Systems and Environment, 6, 799-810. Recuperado de https://doi.org/10.1007/s40808-019-00707-7

Izuka, S. K., Engott, J. A., Rotzoll, K., Bassiouni, M., Johnson, A. G., ..., \& Mair, A. (2018). Volcanic aquifers of Hawai'i - Hydrogeology, water budgets, and conceptual models (U.S. Geological Survey Scientific 
Investigations No. Report 2015-5164). Reston, Virginia, USA: U.S. Geological Survey.

Jasechko, S., \& Taylor, R. G. (2015). Intensive rainfall recharges tropical groundwaters. Environmental Research Letters, 10(12), 124015. Recuperado de https://doi.org/10.1088/1748-9326/10/12/124015

Kendy, E., Zhang, Y., Liu, C., Wang, J., \& Steenhuis, T. (2004). Groundwater recharge from irrigated cropland in the North China Plain: Case study of Luancheng County, Hebei Province, 1949-2000. Hydrological Processes, 18(12), 2289-2302. Recuperado de https://doi.org/10.1002/hyp.5529

Lerner, D. N. (2002). Identifying and quantifying urban recharge: A review. Hydrogeology Journal, 10(1), 143-152. Recuperado de https://doi.org/10.1007/s10040-001-0177-1

Lerner, D. N. (1990). Groundwater recharge in urban areas. Hydrological Process and Water Management in Urban Areas, IAHS, Duisberg, 198, 59-66.

Lewis, F. M., \& Walker, G. R. (2002). Assessing the potential for significant and episodic recharge in Southwestern Australia using rainfall data. Hydrogeology Journal, 10(1), 229-37. Recuperado de https://doi: $10.1007 / \mathrm{s} 10040-001-0172-6$

Lexa, J., Šebesta, J., Chavez, J. A., Hernández, W., \& Pécskay, Z. (2012). Geology and volcanic evolution in the southern part of the San 
Salvador Metropolitan Area. Journal of Geosciences, 56(1), 106140. Recuperado de https://doi.org/10.3190/jgeosci.088

Lihe, Y., Hu, G., Huang, J., Wen, D., Dong, J., Wang, X., \& Li, H. (2011). Groundwater-recharge estimation in the Ordos Plateau, China: Comparison of methods. Hydrogeology Journal, 19(8), 1563-1575. Recuperado de https://doi.org/10.1007/s10040-011-0777-3

Lihe, Y., Guangcai, H., Zhengping, T., \& Ying, L. (2010). Origin and recharge estimates of groundwater in the ordos plateau, People's Republic of China. Environmental Earth Sciences, 60(8), 17311738. Recuperado de https://doi.org/10.1007/s12665-009-0310-3

Mair, A., Hagedorn, B., Tillery, S., El-Kadi, A. I., Westenbroek, S., Ha, K., \& Koh, G.-W. (2013). Temporal and spatial variability of groundwater recharge on Jeju Island, Korea. Journal of Hydrology, 501, 213-226. Recuperado de https://doi.org/10.1016/j.jhydrol.2013.08.015

Manna, F., Murray, S., Abbey, D., Martin, P., Cherry, J., \& Parker, B. (2019). Spatial and temporal variability of groundwater recharge in a sandstone aquifer in a semiarid region. Hydrology and Earth System Sciences, 23(4), 2187-2205. Recuperado de https://doi.org/10.5194/hess-23-2187-2019

MARN, Ministerio del Medio Ambiente y Recursos Naturales. (2017). Directrices para la zonificación ambiental y los usos del suelo para el volcán de San Salvador y zonas aledañas, Órgano Ejecutivo en el 
Ramo de Medio Ambiente y Recursos Naturales. San Salvador, El Salvador: Ministerio del Medio Ambiente y Recursos Naturales.

MARN, Ministerio del Medio Ambiente y Recursos Naturales. (2016a). Plan Nacional de Gestión Integrada del Recuso Hídrico de El Salvador, con énfasis en zonas prioritarias. San Salvador, El Salvador: Ministerio de Medio Ambiente y Recursos Naturales.

MARN, Ministerio del Medio Ambiente y Recursos Naturales. (2016b). Registro de monitoreo hidrometeorológico MARN (datos crudos). San Salvador, El Salvador: Ministerio de Medio Ambiente y Recursos Naturales.

MARN \& OPAMSS, Ministerio del Medio Ambiente y Recursos Naturales \& Oficina de Planificación del Área Metropolitana de San Salvador. (2012). Mapa de uso de suelos de la Subregión Metropolitana de San Salvador. Esc. 1:25,000. San Salvador, El Salvador: Ministerio de Medio Ambiente y Recursos Naturales.

Marrero-Diaz, R., Alcalá, F., Pérez, N., López, D., Melián, G., Padrón, E., \& Padilla, G. (2015). Aquifer recharge estimation through atmospheric chloride mass balance at Las Cañadas Caldera, Tenerife, Canary Islands, Spain. Water, 7(12), 2451-2471. Recuperado de https://doi.org/10.3390/w7052451

McLean, J., Cleveland, S. B., Rotzoll, K., Izuka, S. K., Leigh, J., \& Jacobs, G. A. (2019). The "Ike Wai Hawai" Groundwater Recharge Tool. Gateways 2019, San Diego, CA, USA. 
Naranjo, G., Cruz-Fuentes, T., Cabrera, Ma. C., \& Custodio, E. (2015). Estimating natural recharge by means of chloride mass balance in a volcanic aquifer: Northeastern Gran Canaria (Canary Islands, Spain). Water, 7, 2555-2574. Recuperado de https://doi.org/w10.3390/7062555

Neal, C., \& Kirchner, J. (2000). Sodium and chloride levels in rainfall, mist, stream water and groundwater at the plynlimon catchments, mid-wales: Inferences on Hydrological and Chemical Controls. Hydrology and Earth System Sciences, 4(2), 295-310. Recuperado de https://doi: 10.5194/hess-4-295-2000

Nemaxwi, P., Odiyo, J. O., \& Makungo, R. (2019). Estimation of groundwater recharge response from rainfall events in a semi-arid fractured aquifer: Case study of quaternary catchment $\mathrm{A} 91 \mathrm{H}$, Limpopo Province, South Africa. Cogent Engineering, 6(1), 1636815. Recuperado de https://doi.org/10.1080/23311916.2019.1635815

Ng, G. H. C., McLaughlin, D., Entekhabi, D., \& Scanlon, B. R. (2010). Probabilistic analysis of the effects of climate change on groundwater recharge: Effects of climate change on groundwater recharge. Water Resources Research, 46(1), W07502. Recuperado de https://doi.org/10.1029/2009WR007904

NRCS \& ARS, National Resources Conservation Service \& Agricultural Research Service. (2003). Small Watershed Hydrology: WinTR-55 
User Guide. Washington, DC, USA: / U.S. Department of Agriculture.

PNUD, Programa de las Naciones Unidas para el Desarrollo. (1972). Estudio sobre las aguas subterráneas en la zona metropolitana de San Salvador (no publicado). San Salvador, El Salvador: Programa de las Naciones Unidas para el Desarrollo.

Rman, N. (2016). Hydrogeochemical and isotopic tracers for identification of seasonal and long-term over-exploitation of the Pleistocene thermal waters. Environmental Monitoring and Assessment, 188(4), 242. Recuperado de https://doi.org/10.1007/s10661-016-5250-2

Rew, R., Davis, G., Emmerson, S., Cormack, C., Caron, J., Pincus, R., ..., \& Fisher, W. (1989). Unidata NetCDF. UCAR/NCAR - Unidata. Recuperado de https://doi.org/10.5065/D6H70CW6

Reynolds, J. H. (1980). Late Tertiary Volcanic Stratigraphy of Northern Central America. Bulletin of Volcanology, 43(3), 601-607.

Rico, M. A., Bourne, W. C., \& Menéndez, M. E. (1965). Levantamiento General de Suelos de la República de El Salvador. Mapa Esc. 1:50,000. San Salvador, El Salvador: Dirección General de Investigaciones Agronómicas.

Rushton, K. R., \& Ward, C. (1979). The estimation of groundwater recharge. Journal of Hydrology, 41(3-4), 345-361. Recuperado de https://doi.org/10.1016/0022-1694(79)90070-2 
Scanlon, B. R., Keese, K. E., Flint, A. L., Flint, L. E., Gaye, C. B., Edmunds, W. M., \& Simmers, I. (2006). Global synthesis of groundwater recharge in semiarid and arid regions. Hydrological Processes, 20(15), 3335-3370. Recuperado de https://doi.org/10.1002/hyp.6335

Scanlon, B. R., Healy, R. W., \& Cook, P. G. (2002). Choosing appropriate techniques for quantifying groundwater recharge. Hydrogeology Journal, 10, 18-39.

Schmidt-Thomé, M. (1975). The geology in the San Salvador area (El Salvador, Central America), a basis for city development and planning. Geologisches Jahrbuch, 13, 207-228.

Schosinsky, G. (2006). Cálculo de la recarga potencial de acuíferos mediante un balance hídrico de suelos. Revista Geológica América Central, 34(35), 13-30.

Schulzweida, U. (2019). CDO, Climate Data Operator, User Guide (version 1.9.8) (software). Recuperado de https://doi.org/10.5281/zenodo.3539275

Seiler, K.-P., \& Gat, J.R. (2007). Groundwater recharge from run-off, infiltration and percolation, Water science and technology library. Dordrecht, The Netherlands: Springer.

Servicio Hidrológico Nacional. (2005). Balance hídrico integrado y dinámico de El Salvador. Componente Evaluación de Recursos Hídricos. San Salvador, El Salvador: Servicio Nacional de Estudios Territoriales, Servicio Hidrológico Nacional. 
Siles, P., Vaast, P., Dreyer, E., \& Harmand, J.-M. (2010). Rainfall partitioning into throughfall, stemflow and interception loss in a coffee (Coffea arabica L.) monoculture compared to an agroforestry system with Inga densiflora. Journal of Hydrology, 395(1), 39-48. Recuperado de https://doi.org/10.1016/j.jhydrol.2010.10.005

Sophocleous, M. (1993). Comparative review and synthesis of groundwater recharge estimates for the Great Bend Prairie aquifer of Kansas. Current Research on Kansas Geological Survey, 235, 4154.

Stanton, J. S., Ryter, D. W., \& Peterson, S. M. (2012). Effects of linking a soil-water-balance model with a groundwater-flow model. Ground Water Journal, 51(4), 613-622. Recuperado de https://doi.org/10.1111/j.1745-6584.2012.01000.x

Steenhuis, T. S., \& Van-Der-Molen, W. H. (1986). The ThornthwaiteMather procedure as a simple engineering method to predict recharge. Journal of Hydrology, 84(3-4), 221-229. Recuperado de https://doi.org/10.1016/0022-1694(86)90124-1

Thornthwaite, C. W. (1948). An approach toward a rational classification of climate. Geographical Review, 38(1), 55-94. Recuperado de https://doi.org/10.2307/210739

Thornthwaite, C. W., \& Mather, J. R. (1957). Instructions and tables for computing potential evapotranspiration and the water balance. Publications in Climatology, 10(3), 185-243. 
Thornthwaite, C. W., \& Mather, J. R. (1955). The water balance. Publications in Climatology, 8(1), 1-104.

Ting, C. S., Kerh, T., \& Liao, C. J. (1998). Estimation of groundwater recharge using the chloride mass-balance method, Pingtung Plain, Taiwan. Hydrogeology Journal, 6(2), 282-292.

Weber, H. S., Wisemann, G., \& Wittekindt, H. (1974). Geologische Übersichtskarte der Republik El Salvador. Esc. 1:100,000. San Salvador, El Salvador: Instituto Geográfico. Nacional, Ing. Pablo Arnoldo Guzmán.

Westenbroek, S. M., Engott, J. A., Kelson, V. A., \& Hunt, R. J. (2018). SWB Version 2.0 (software) -A soil-water-balance code for estimating net infiltration and other water-budget components (Report No. 6-A59), Techniques and Methods. Reston, USA. Recuperado de https://doi.org/10.3133/tm6A59

Westenbroek, S. M., Kelson, V. A., Dripps, W. R., Hunt, R. J., \& Bradbury, K. R. (2010). SWB - A Modified Thornthwaite-Mather Soil-WaterBalance Code for Estimating Groundwater Recharge (6-A31). Reston, USA: U.S. Geological Survey Techniques and Methods.

Williams, H., \& Meyer-Abich, H. (1955). Volcanism in the southern part of El Salvador with particular reference to the collapse basins of Coatepeque and Ilopango. University of California Publications in Geology Sciences, 32, 1-64. 
Woodward, D. E., Hawkins, R. H., Jiang, R., Hjelmfelt, Jr., A. T., VanMullem, J. A., \& Quan, Q. D. (2003). Runoff curve number method: examination of the initial abstraction ratio. In: World Water \&amp; Environmental Resources Congress 2003. World Water and Environmental Resources Congress 2003, American Society of Civil Engineers, Philadelphia, Pennsylvania, United States (pp. 1-10). Recuperado de https://doi.org/10.1061/40685(2003)308 